\title{
INTERPOLATING SEQUENCES FOR WEIGHTED SPACES OF ANALYTIC FUNCTIONS ON THE UNIT BALL OF A HILBERT SPACE
}

\author{
OSCAR BLASCO*, PABLO GALINDO ${ }^{\dagger}$, MIKAEL LINDSTRÖM $^{* *}$, AND ALEJANDRO MIRALLES A $^{\ddagger}$
}

\begin{abstract}
We show that an interpolating sequence for the weighted Banach space of analytic functions on the unit ball of a Hilbert space is hyperbolically separated. In the case of the so-called standard weights, a sufficient condition for a sequence to be linear interpolating is given in terms of Carleson type measures. Other conditions to be linearly interpolating are provided as well. Our results apply to the space of Bloch functions of such unit ball.
\end{abstract}

\section{Introduction AND PRELIMINARIES}

Throughout the paper $E$ stands for a complex Hilbert space of arbitrary dimension and $B_{E}=\{x \in E:\|x\|<1\}$ for its open unit ball. Let $v: B_{E} \rightarrow(0, \infty)$ be a weight, that is, a continuous positive function. The weighted space of analytic functions

$$
H_{v}^{\infty}\left(B_{E}\right):=\left\{f: B_{E} \rightarrow \mathbb{C}: f \text { is analytic and }\|f\|_{v}=\sup _{x \in B_{E}} v(x)|f(x)|<\infty\right\}
$$

is a Banach space when endowed with the $\|\cdot\|_{v}$ norm. Here we are mostly interested in the standard weights $v_{\alpha}(x)=\left(1-\|x\|^{2}\right)^{\alpha}, \alpha \geq 0$. When $\alpha=0$ we get the infinite dimensional generalization $H^{\infty}\left(B_{E}\right)$ of the Hardy algebra $H^{\infty}$ of the unit disc. Nevertheless, some of our results hold true for more general weights.

An analytic function $f: B_{E} \rightarrow \mathbb{C}$ is said to belong to the Bloch space $\mathcal{B}\left(B_{E}\right)$ if $\sup _{x \in B_{E}}(1-$ $\left.\|x\|^{2}\right)\|\nabla f(x)\|<\infty$ or, equivalently, if $\sup _{x \in B_{E}}\left(1-\|x\|^{2}\right)|R f(x)|<\infty$, where $R f(x)$ is the radial derivative of $f$ at $x, R f(x)=\langle x, \overline{\nabla f(x)}\rangle$. Both suprema define equivalent Banach space norms - modulo the constant functions- in $\mathcal{B}\left(B_{E}\right)$. We will use both the norms

$$
\|f\|_{\mathcal{B}\left(B_{E}\right)}:=|f(0)|+\sup _{x \in B_{E}}\left(1-\|x\|^{2}\right)\|\nabla f(x)\|
$$

and

$$
\|f\|_{\mathcal{R}\left(B_{E}\right)}:=|f(0)|+\sup _{x \in B_{E}}\left(1-\|x\|^{2}\right)|R f(x)| .
$$

2010 Mathematics Subject Classification. Primary 30D45, 46E50. Secondary 46G20.

Key words and phrases. interpolating sequence, hyperbolically separated, Bloch function in the ball, infinite dimensional holomorphy, weighted space of analytic functions.

${ }^{*}$ Partially supported by MTM2014-53009-P .

${ }^{\dagger}$ Partially supported by MTM2014-53241-P .

**Partially supported by MTM2014-53241-P and the Academy of Finland project 296718.

‡Partially supported by MTM2014-53241-P, P1-1B2014-35 and AICO/2016/030. 
We shall deal also with the following generalization: for $\alpha>0$, it is said that $f \in \mathcal{B}^{\alpha}\left(B_{E}\right)$ whenever $\sup _{x \in B_{E}}\left(1-\|x\|^{2}\right)^{\alpha}|R f(x)|<\infty$ and we write

$$
\|f\|_{\mathcal{R}^{\alpha}\left(B_{E}\right)}:=|f(0)|+\sup _{x \in B_{E}}\left(1-\|x\|^{2}\right)^{\alpha}|R f(x)| .
$$

The space of Bloch functions on the unit ball of a Hilbert space was introduced and studied in [3] and its generalization $\mathcal{B}^{\alpha}\left(B_{E}\right)$ has been considered in [17] and [30]. If $E=\mathbb{C}$, we get the classical Bloch-type spaces denoted by $\mathcal{B}^{\alpha}$. We shall use the notation $\mathcal{B}^{\alpha}\left(B_{E}\right)_{0}:=\{f \in$ $\left.\mathcal{B}^{\alpha}\left(B_{E}\right): f(0)=0\right\}$ and $H_{v}^{\infty}\left(B_{E}\right)_{0}:=\left\{f \in H_{v}^{\infty}\left(B_{E}\right): f(0)=0\right\}$.

The aim of this article is to study interpolating sequences for these spaces. We will say that $\left(w_{n}\right) \subset B_{E}$ is interpolating for $H_{v}^{\infty}\left(B_{E}\right)$ if the mapping

$$
S: H_{v}^{\infty}\left(B_{E}\right) \rightarrow \ell_{\infty} \text { defined by } S f=\left(v\left(w_{n}\right) f\left(w_{n}\right)\right)
$$

is onto. As usual we write $\left\|\left(\alpha_{n}\right)\right\|=\sup _{n}\left|\alpha_{n}\right|$ for any bounded sequence of complex numbers. A sequence $\left(w_{n}\right) \subset B_{E} \backslash\{0\}$ is said to be interpolating (for the radial derivative) for the Bloch space $\mathcal{B}^{\alpha}\left(B_{E}\right)$, if the mapping

$$
S: \mathcal{B}^{\alpha}\left(B_{E}\right) \rightarrow \ell_{\infty} \text { defined by } S(f)=\left(\left(1-\left\|w_{n}\right\|^{2}\right)^{\alpha} R f\left(w_{n}\right)\right)
$$

is onto. Notice that 0 cannot be included into the interpolating sequence since $R f(0)=0$.

In case $S$ has a linear right inverse, we say that $\left(w_{n}\right)$ is a linear interpolating sequence.

For a given interpolating sequence $\left(w_{n}\right)$, any constant $M>0$ such that whenever $\alpha \in \ell_{\infty}$ there exists $f$ with $S(f)=\alpha$ and $\|f\| \leq M\|\alpha\|_{\infty}$ is called an interpolation constant. Such constants exist by the Open Mapping Theorem.

There are in the mathematical literature several meanings for the expression "interpolating sequence" for $\mathcal{B}$ that do not take into account the derivative of the function. See [5] and [29] for details on different types of interpolation for $\mathcal{B}$. For further information on interpolating sequences on spaces of analytic functions we refer to [26].

After the celebrated result by L. Carleson which characterizes interpolating sequences for the space $H^{\infty}$ (see [14]) also interpolating sequences for the spaces $H_{v_{\alpha}}^{\infty}$ were characterized by K. Seip in [25]. In connection with Hankel operators, K. R. M. Attele in [1] studied in connection with Hankel operators interpolating sequences for the derivatives in the Bloch space $\mathcal{B}$. Such sequences were used by K. Madigan and A. Matheson [18] as a tool for the study of compactness of some composition operators. The study of interpolating sequences for Banach spaces of analytic functions on $\mathbb{B}_{n}$, the open unit ball of the Euclidean space $\mathbb{C}^{n}$, was initiated by B. Berndtsson in [2] for $H^{\infty}\left(\mathbb{B}_{n}\right)$ and by X. Massaneda in [19] for $H_{v_{\alpha}}^{\infty}\left(\mathbb{B}_{n}\right)$ with $\alpha>0$. They found some necessary and sufficient conditions for a sequence to be interpolating in those spaces. Interpolating sequences in the $n$-ball for the so-called fractional derivatives were investigated in [7]. The existence of interpolating sequences for a given Banach function space shows an abundance of elements in the space and it has turned to be very useful in the study of weighted composition operators. The study of interpolating sequences for $H^{\infty}\left(B_{E}\right)$ was initiated in [11] and $[12]$ (see also [22]).

In this paper we prove that being hyperbolically separated is also a necessary condition for a sequence to be interpolating in $\mathcal{B}^{\alpha}\left(B_{E}\right)$ or in $H_{v}^{\infty}\left(B_{E}\right)$ for quite general weights. In Section 3 we provide some explicit and enlightening examples of interpolating sequences. We also show that if the sequence is interpolating for $H^{\infty}\left(B_{E}\right)$, then it is linear interpolating for $\mathcal{B}^{\alpha}\left(B_{E}\right)$ as 
well as for $H_{v_{\alpha}}^{\infty}\left(B_{E}\right)$. By using a suitable extension of the notion of Carleson measure, we obtain a sufficient condition for a sequence to be linear interpolating in $H_{v_{\alpha}}^{\infty}\left(B_{E}\right)$ (Theorem 5.7). This result extends to $H_{v_{\alpha}}^{\infty}\left(B_{E}\right)$ and $\mathcal{B}^{\alpha}\left(B_{E}\right)$ the previously known results (see [1] and [18]) in the unit disc stating that a sufficiently separated sequence is interpolating. In Section 7 we present a number of results concerning $\mathcal{B}\left(B_{E}\right)$ that are extension of the classical ones.

For background on analytic functions on subsets of Hilbert or more general Banach spaces, we refer to [20]. We will use, frequently without further notice, the following consequence of Montel's theorem ([20, Proposition 9.16]).

Lemma 1.1. If $F_{m} \in H\left(B_{E}\right)$, the series $\sum_{m} F_{m}$ is pointwise convergent and the series has uniformly bounded partial sums on compact subsets of $B_{E}$, then it defines an analytic function.

A crucial tool in the study of analytic functions on the unit ball of a Hilbert space is the homogeneity of the ball. Specifically, the existence of the Möbius transforms $\varphi_{a}: B_{E} \rightarrow B_{E}$ for each $a \in B_{E}$ defined by

$$
\varphi_{a}(x)=\left(s_{a} Q_{a}+P_{a}\right)\left(m_{a}(x)\right)
$$

where $s_{a}=\sqrt{1-\|a\|^{2}}, m_{a}: B_{E} \rightarrow B_{E}$ is the analytic function

$$
m_{a}(x)=\frac{a-x}{1-\langle x, a\rangle}
$$

and $P_{a}=\frac{1}{\|a\|^{2}} a \otimes a$ where $u \otimes v(x)=\langle x, u\rangle v$ and $Q_{a}=I d-P_{a}$ are the orthogonal projection on the one dimensional subspace generated by $a$ and on its orthogonal complement respectively. We recall that $\varphi_{a} \circ \varphi_{a}(x)=x$ and $\varphi_{a}(a)=0$.

The pseudo-hyperbolic metric on $B_{E}$ is defined by $\rho_{E}(x, y):=\left\|\varphi_{x}(y)\right\|$. In case $E=\mathbb{C}$, we write $\rho$ instead of $\rho_{\mathbb{C}}$. The pseudo-hyperbolic disc given by $\left\{y \in B_{E}: \rho_{E}(x, y)<R\right\}$ is denoted by $D(x, R)$. We recall some facts to be used later (see [16] p. 99) Let $x \in B_{E}$ and $R \in(0,1)$. Then

$$
\rho_{E}(x, y)^{2}=\left\|\varphi_{x}(y)\right\|^{2}=1-\frac{\left(1-\|x\|^{2}\right)\left(1-\|y\|^{2}\right)}{|1-\langle x, y\rangle|^{2}}, \quad y \in B_{E} .
$$

and

$$
\frac{1-R^{2}}{4}\left(1-\|x\|^{2}\right) \leq 1-\|y\|^{2} \leq \frac{4}{1-R^{2}}\left(1-\|x\|^{2}\right), \quad y \in D(x, R) .
$$

We shall write $\mathbb{B}_{n}$ for the unit ball of $\mathbb{C}^{n}$ and denote by $\nu_{n}$ the normalized measure in $\mathbb{B}_{n}$ for $n \geq 1$. It is well known that for $n=1$ the pseudo-hyperbolic disc $D(z, R)$ becomes an Euclidean disc while in the case $n \geq 2$ it is not an Euclidean disc (unless $z=0$ ) but an ellipsoid (see $\left[24\right.$, pages 29,30]) and the value $\nu_{n}(D(z, R))$ is

$$
\nu_{n}(D(z, R))=R^{2 n}\left(\frac{1-|z|^{2}}{1-R^{2}|z|^{2}}\right)^{n+1}, \quad z \in \mathbb{B}_{n}, 0<R<1 .
$$

A sequence $\left(w_{n}\right) \subset B_{E}$ is said to be hyperbolically $r$-separated for $r>0$ if $\rho_{E}\left(w_{n}, w_{m}\right)>$ $r$ for $n \neq m$. We say that the sequence is hyperbolically separated if it is hyperbolically $r$-separated for some $r>0$. We will say that the sequence $\left(w_{n}\right)$ satisfies the Carleson's condition if $\prod_{m \neq n} \rho_{E}\left(w_{n}, w_{m}\right) \geq \delta$ for some $\delta$ and for all $m \in \mathbb{N}$. 


\section{Carleson measures on the unit ball $B_{E}$}

For any $\xi \in E,\|\xi\|=1$ and $0<h<1$ we shall denote by $S(\xi, h)$ the Carleson window given by

$$
S(\xi, h)=\left\{y \in B_{E}:|1-\langle y, \xi\rangle|<2 h\right\} .
$$

We write $S(\xi, h)=B_{E}$ for $h \geq 1$.

Definition 2.1. Let $\eta$ be a finite Borel measure on $B_{H}$ and $\beta>0$. We say that $\eta$ is a $\beta$-Carleson measure whenever there exists $C>0$ such that

$$
\eta(S(\xi, h)) \leq C h^{\beta}, \quad \text { for all }\|\xi\|=1, \text { and all } 0<h<1
$$

We write $\|\eta\|_{\beta}=\sup _{\|\xi\|=1,0<h<1} \frac{\eta(S(\xi, h))}{h^{\beta}}$.

Lemma 2.2. Let $\eta$ be a finite Borel measure on $B_{H}$ and $\beta>0$. Define for $\alpha>0$

$$
I_{\eta}(\alpha, \beta)=\sup _{\|x\|<1} \frac{1}{\left(1-\|x\|^{2}\right)^{\beta}} \int_{B_{E}}\left(\frac{\mid 1-\|x\|^{2}}{|1-\langle w, x\rangle|}\right)^{\alpha} d \eta(w) \in[0, \infty] .
$$

(i) If $I_{\eta}(\alpha, \beta)<\infty$ for some $\alpha>0$, then $\eta$ is a $\beta$-Carleson measure.

(ii) If $\eta$ is a $\beta$-Carleson measure, then $I_{\eta}(\alpha, \beta)<\infty$ for any $\alpha>\beta$.

Furthermore, for $\alpha>\beta$ one has

$$
\frac{2^{\alpha}-2^{\beta}}{2^{2 \alpha}} I_{\eta}(\alpha, \beta) \leq\|\eta\|_{\beta} \leq 2^{\beta} 3^{\alpha} I_{\eta}(\alpha, \beta) .
$$

Proof. (i) Let $0<h<1$ and $\xi \in B_{E}$ with $\|\xi\|=1$ and set $x=(1-h) \xi$. Hence $h=1-\|x\|$ and for $y \in S(\xi, h)$ we have

$$
|1-\langle y, x\rangle|=|1-\langle y, \xi\rangle+h\langle y, \xi\rangle| \leq 3 h \leq 3\left(1-\|x\|^{2}\right)
$$

Therefore

$$
\begin{aligned}
\eta(S(\xi, h)) & =\int_{S(\xi, h)} d \eta(y) \\
& \leq 3^{\alpha} \int_{B_{E}}\left(\frac{\mid 1-\|x\|^{2}}{|1-\langle y, x\rangle|}\right)^{\alpha} d \eta(y) \\
& \leq 3^{\alpha} I_{\eta}(\alpha, \beta)\left(1-\|x\|^{2}\right)^{\beta} \leq 3^{\alpha} I_{\eta}(\alpha, \beta) 2^{\beta} h^{\beta} .
\end{aligned}
$$

(ii) Let $\alpha>\beta$. For each $x \in B_{E}, x \neq 0$, we write $\xi=\frac{x}{\|x\|}$. Note that if $y \notin S(\xi, h)$ and $h>\frac{1-\|x\|}{2}$, then

$$
\begin{aligned}
|1-\langle y, x\rangle| & \geq|1-\langle y, \xi\rangle|-|\langle y, x-\xi\rangle| \\
& \geq 2 h-\left\|\frac{x}{\|x\|}-x\right\|\|y\| \\
& \geq 2 h-(1-\|x\|)
\end{aligned}
$$

Define $E_{0}=S\left(\xi, 1-\|x\|^{2}\right)$ and

$$
E_{k}=S\left(\xi, 2^{k}\left(1-\|x\|^{2}\right)\right) \backslash S\left(\xi, 2^{k-1}\left(1-\|x\|^{2}\right)\right) .
$$


INTERPOLATING SEQUENCES FOR WEIGHTED SPACES OF ANALYTIC FUNCTIONS

Now for $k \geq 1, y \in E_{k}$ and selecting $h=2^{k-1}\left(1-\|x\|^{2}\right)$ in the above estimate, we obtain that

$$
|1-\langle y, x\rangle| \geq 2^{k}\left(1-\|x\|^{2}\right)-(1-\|x\|) \geq 2^{k-1}\left(1-\|x\|^{2}\right) .
$$

Select $M \in \mathbb{N}$ so that $2^{M-1}\left(1-\|x\|^{2}\right)<1 \leq 2^{M}\left(1-\|x\|^{2}\right)$. Hence

$$
\begin{aligned}
\int_{B_{E}} \frac{\left(\mid 1-\|x\|^{2}\right)^{\alpha}}{|1-\langle w, x\rangle|^{\alpha}} d \eta(w) & =\sum_{k=0}^{M} \int_{E_{k}} \frac{\left(\mid 1-\|x\|^{2}\right)^{\alpha}}{|1-\langle w, x\rangle|^{\alpha}} d \eta(w) \\
& \leq 2^{\alpha} \eta\left(S\left(\xi, 1-\|x\|^{2}\right)+\sum_{k=1}^{M} 2^{-\alpha(k-1)} \eta\left(S\left(\xi, 2^{k}\left(1-\|x\|^{2}\right)\right)\right.\right. \\
& \leq 2^{\alpha}\|\eta\|_{\beta}\left(1-\|x\|^{2}\right)^{\beta}\left(\sum_{k=0}^{\infty} 2^{k(\beta-\alpha)}\right) \\
& =\frac{2^{2 \alpha}\|\eta\|_{\beta}}{2^{\alpha}-2^{\beta}}\left(1-\|x\|^{2}\right)^{\beta}
\end{aligned}
$$

This completes the proof and give the stated estimates.

Recall that for $x \in B_{E}$, the evaluation map $\delta_{x}$ is given by $\delta_{x}(f)=f(x)$ for $f \in H\left(B_{E}\right)$.

Definition 2.3. Given $\left(w_{n}\right)_{n=1}^{\infty} \subset B_{E}$ and $\gamma>0$ we define

$$
\eta_{\gamma,\left(w_{n}\right)}=\sum_{n=1}^{\infty}\left(1-\left\|w_{n}\right\|^{2}\right)^{\gamma} \delta_{w_{n}} .
$$

In particular $\eta_{\gamma,\left(w_{n}\right)}\left(B_{E}\right)<\infty$ if and only if $\sum_{n=1}^{\infty}\left(1-\left\|w_{n}\right\|^{2}\right)^{\gamma}<\infty$.

The following characterization is a direct consequence of Lemma 2.2 together with (1.2).

Lemma 2.4. Let $\left(w_{n}\right)_{n=1}^{\infty} \subset B_{E}$ and $\alpha>\beta>0$. Then for each $\gamma>0$ the measure $\eta_{\gamma,\left(w_{n}\right)}$ is a $\beta$-Carleson measure if and only if there exists $C>0$ such that

$$
\sum_{n \in \mathbb{N}}\left(1-\rho_{E}^{2}\left(x, w_{n}\right)\right)^{\alpha / 2}\left(1-\left\|w_{n}\right\|^{2}\right)^{\gamma-\alpha / 2} \leq C\left(1-\|x\|^{2}\right)^{\beta-\alpha / 2}, \quad x \in B_{E} .
$$

In particular, the following are equivalent:

(i) $\eta_{\beta,\left(w_{n}\right)}$ is a $\beta$-Carleson measure.

(ii) $I_{\eta_{\beta,\left(w_{n}\right)}}(\alpha, \beta)=\sup _{\|x\|<1} \sum_{n \in \mathbb{N}}\left(1-\rho_{E}\left(x, w_{n}\right)^{2}\right)^{\alpha / 2}\left(\frac{1-\left\|w_{n}\right\|^{2}}{1-\|x\|^{2}}\right)^{\beta-\alpha / 2}<\infty$.

(iii) $I_{\eta_{\beta,\left(w_{n}\right)}}(\alpha, \beta)=\sup _{\|x\|<1} \sum_{n \in \mathbb{N}} \frac{\left(1-\left\|w_{n}\right\|^{2}\right)^{\beta}\left(1-\|x\|^{2}\right)^{\alpha-\beta}}{\left|1-\left\langle x, w_{n}\right\rangle\right|^{\alpha}}<\infty$.

We consider the following notation introduced in [19] for $\mathbb{B}_{n}$. For $p, q>0$ and $\left(w_{j}\right) \subset B_{E}$, we denote

$$
K\left(\left\{w_{j}\right\}, p, q\right)=\sup _{k \in \mathbb{N}} \sum_{j \neq k} \frac{\left(1-\left\|w_{k}\right\|^{2}\right)^{p}\left(1-\left\|w_{j}\right\|^{2}\right)^{q}}{\left|1-\left\langle w_{k}, w_{j}\right\rangle\right|^{p+q}} .
$$

Corollary 2.5. Let $\gamma, \beta>0$ and $\left(w_{j}\right) \subset B_{E}$. If $\eta_{\beta,\left(w_{j}\right)}$ is a $\beta$-Carleson measure then

$$
K\left(\left\{w_{j}\right\}, \gamma, \beta\right) \leq I_{\eta_{\beta,\left(w_{j}\right)}}(\beta+\gamma, \beta)<\infty .
$$


Remark 2.6. It was shown in $\left[19\right.$, Lemma 1.4] that for $E=\left(\mathbb{C}^{n},\|\cdot\|_{2}\right)$ one has that $\eta_{\beta,\left(z_{j}\right)}$ is a $\beta$-Carleson measure for $\beta \geq n$ if and only if $K\left(\left\{z_{j}\right\}, \gamma, \beta\right)<\infty$ for any $0<\gamma \leq \beta$.

Let us now relate the notion of "hyperbolic separation" with $\beta$-Carleson measures. Combining Remark 2.6 and [19, Lemma 1.5] it was shown that any hyperbolically separated sequence $\left(z_{j}\right) \subset \mathbb{B}_{n}$ satisfies that $\eta_{\beta,\left(z_{j}\right)}$ is a $\beta$-Carleson measure for $\beta>n$. We shall give an alternative proof of such a result using the following well known estimate (see [28, Lemma 2.24])

$$
|f(z)|^{p} \leq \frac{C}{\left(1-|z|^{2}\right)^{n+1}} \int_{D(z, r)}|f(w)|^{p} d \nu_{n}(w), \quad z \in \mathbb{B}_{n} .
$$

for any $0<r<1,0<p<\infty$ and any holomorphic function $f$ in $\mathbb{B}_{n}$.

Proposition 2.7. Let $n \in \mathbb{N}$ and let $\left(z_{j}\right) \subset \mathbb{B}_{n}$ be a hyperbolically separated sequence, then $\eta_{\beta,\left(z_{j}\right)}$ is a $\beta$-Carleson measure for any $\beta>n$.

Proof. Assume that $\rho_{\mathbb{C}^{n}}\left(z_{j}, z_{k}\right) \geq 2 R$ for $j \neq k$. Hence $D\left(z_{j}, R\right)$ are pairwise disjoint sets in $\mathbb{B}_{n}$. Let us show that $\left(z_{j}\right)$ satisfies (iii) in Lemma 2.4 for any $\beta>n$ and any $\alpha>\beta$. Let $z \in \mathbb{B}_{n}$. Applying (2.5) to the function $\frac{1}{(1-\langle w, z\rangle)^{\alpha}}$ for the point $z_{j} \in D\left(z_{j}, R\right)$ together with (1.3), we have

$$
\begin{aligned}
\sum_{j \in \mathbb{N}} \frac{\left(1-\left|z_{j}\right|^{2}\right)^{\beta}\left(1-|z|^{2}\right)^{\alpha-\beta}}{\left|1-\left\langle z_{j}, z\right\rangle\right|^{\alpha}} & \leq C_{R}\left(1-|z|^{2}\right)^{\alpha-\beta} \sum_{j \in \mathbb{N}} \int_{D\left(z_{j}, R\right)} \frac{\left(1-|w|^{2}\right)^{\beta-n-1}}{|1-\langle w, z\rangle|^{\alpha}} d \nu_{n}(w) \\
& =C_{R}\left(1-|z|^{2}\right)^{\alpha-\beta} \int_{\cup_{j} D\left(z_{j}, R\right)} \frac{\left(1-|w|^{2}\right)^{\beta-n-1}}{|1-\langle w, z\rangle|^{\alpha}} d \nu_{n}(w) .
\end{aligned}
$$

Now use the well-known fact (see [24, Page 18]) that for $c>0$ and $t>-1$

$$
\int_{\mathbb{B}_{n}} \frac{\left(1-|w|^{2}\right)^{t} d \nu_{n}(w)}{|1-\langle w, z\rangle|^{n+1+t+c}} \leq \frac{C}{\left(1-|z|^{2}\right)^{c}} .
$$

In our case $\beta-n-1=t>-1$ and $c=\alpha-\beta>0$, so we get the estimate

$$
\sum_{j \in \mathbb{N}} \frac{\left(1-\left|z_{j}\right|^{2}\right)^{\beta}\left(1-|z|^{2}\right)^{\alpha-\beta}}{\left|1-\left\langle z_{j}, z\right\rangle\right|^{\alpha}} \leq C_{R} C .
$$

Remark 2.8. For the unit ball of infinite dimensional Hilbert spaces the fact that a sequence is hyperbolically separated does not imply that $\eta_{\beta,\left(w_{j}\right)}$ is a $\beta$-Carleson measure for any $\beta>0$. It suffices to use $w_{j}=\frac{1}{2} e_{j}$ which satisfies that $\rho_{E}\left(w_{j}, w_{k}\right)=\frac{\sqrt{7}}{4}$ for $k \neq j$ and $\sum_{j}\left(1-\left\|w_{j}\right\|^{2}\right)^{\beta}=\infty$ for any $\beta>0$.

Invoking now Proposition 2.7, Lemma 2.4 and the fact $\rho(\|x\|,\|y\|) \leq \rho_{E}(x, y)$ for any $x, y \in$ $B_{E}$ we obtain the following result.

Corollary 2.9. Let $\left(w_{j}\right) \subset B_{E}$ such that $\left(\left\|w_{j}\right\|\right) \subset \mathbb{D}$ is a hyperbolically separated sequence. Then $\eta_{\beta,\left(w_{j}\right)}$ is a $\beta$-Carleson measure for any $\beta>1$. 


\section{Examples of InTERPolating SEQUENCES}

Let us start by pointing out that in the case of infinite dimensional Hilbert spaces we can find interpolating sequences for $H_{v}^{\infty}\left(B_{E}\right)$ and in $\mathcal{B}^{\alpha}\left(B_{E}\right)$ whose interpolating functions are polynomials.

Proposition 3.1. Let $\left(e_{n}\right) \subset E$ be an orthonormal sequence and let $u$ be a bounded radial weight on $\mathbb{D}$. Then for each $\left(z_{n}\right) \subset \mathbb{D}$ such that

$$
\left(\inf _{n}\left|z_{n}\right|\right)\left(\inf _{n} u\left(\left|z_{n}\right|\right)\right)>0
$$

the sequence $\left(w_{n}\right)=\left(z_{n} e_{n}\right)$ is linear interpolating for $H_{v}^{\infty}\left(B_{E}\right)$, where $v(x)=u(\|x\|)$.

Specifically, for any bounded sequence $\left(\alpha_{k}\right) \subset \mathbb{C}$ and any degree $d \geq 2$, there is a polynomial $P_{d} \in \mathcal{P}\left({ }^{d} E\right)$ such that $S\left(P_{d}\right)=\left(\alpha_{n}\right)$, where $\mathcal{P}\left({ }^{d} E\right)$ stands for the set of $d$-homogeneous polynomials defined on $B_{E}$ and $S$ is the map defined in (1.1).

Proof. Consider the $d$-homogeneous polynomial

$$
P_{d}(x)=\sum_{n=1}^{\infty} \frac{\alpha_{n}}{u\left(\left|z_{n}\right|\right) z_{n}^{d}}\left\langle x, e_{n}\right\rangle^{d}
$$

Clearly $P_{d}$ is well-defined since $\left(\frac{\alpha_{n}}{z_{n}^{d} v\left(w_{n}\right)}\right) \in \ell_{\infty}$ and $\sum_{n}\left|\left\langle x, e_{n}\right\rangle\right|^{d} \leq\|x\|^{d}$. Note that $u(\|x\|)\left|P_{d}(x)\right| \leq C\left\|\left(\alpha_{n}\right)\right\|_{\infty}$ for $C=\sup _{z \in \mathbb{D}} u(z) \cdot\left(\inf _{n}\left|z_{n}\right|\right)^{-d}\left(\inf _{n} u\left(\left|z_{n}\right|\right)\right)^{-1}$ and $v\left(w_{n}\right) P_{d}\left(w_{n}\right)=$ $\alpha_{n}$ for any $n \in \mathbb{N}$.

Proposition 3.2. Let $\left(e_{n}\right) \subset E$ be an orthonormal sequence and $\alpha>0$. Then for each $\left(z_{n}\right) \subset \mathbb{D}$ such that

$$
0<\inf _{n}\left|z_{n}\right| \leq \sup _{n}\left|z_{n}\right|<1
$$

the sequence $\left(w_{n}\right)=\left(z_{n} e_{n}\right)$ is linear interpolating for $\mathcal{B}^{\alpha}\left(B_{E}\right)$.

Specifically, for any bounded sequence $\left(\alpha_{k}\right) \subset \mathbb{C}$ and any degree $d \geq 2$, there is a polynomial $P_{d} \in \mathcal{P}\left({ }^{d} E\right)$ such that $S\left(P_{d}\right)=\left(\alpha_{n}\right)$.

Proof. We argue similarly to Proposition 3.1 using now

$$
Q_{d}(x)=\sum_{n=1}^{\infty} \frac{\alpha_{n}}{d z_{n}^{d}\left(1-\left|z_{n}\right|^{2}\right)^{\alpha}}\left\langle x, e_{n}\right\rangle^{d} .
$$

Clearly $Q_{d}$ is well-defined since $\ell_{2} \subset \ell_{d}$ and $\left(\frac{\alpha_{n}}{d z_{n}^{d}\left(1-\left|z_{n}\right|^{2}\right)^{\alpha}}\right) \in \ell_{\infty}$ due to (3.2). Since $R Q_{d}(x)=$ $d Q_{d}(x)$ one obtains for each $\|x\|<1$

$$
\left(1-\|x\|^{2}\right)^{\alpha}\left|R Q_{d}(x)\right| \leq\left\|\left(\alpha_{n}\right)\right\| \sum_{n=1}^{\infty} \frac{\left(1-\|x\|^{2}\right)^{\alpha}}{\left|z_{n}\right|^{d}\left(1-\left|z_{n}\right|^{2}\right)^{\alpha}}\left|\left\langle x, e_{n}\right\rangle\right|^{d} \leq C\left\|\left(\alpha_{n}\right)\right\|\|x\|^{d} .
$$

On the other hand $\left(1-\left\|w_{n}\right\|^{2}\right)^{\alpha} R Q_{d}\left(w_{n}\right)=\alpha_{n}$ for any $n \in \mathbb{N}$.

Let us give another procedure to generate interpolating sequences where the interpolating functions are explicitly given. 
Proposition 3.3. Let $\alpha>0$ and $\left(z_{n}\right) \subset \mathbb{D}$ such that $\inf _{n}\left|z_{n}\right|>0$. For any orthonormal sequence $\left(e_{n}\right) \subset E$, denote $w_{n}=z_{n} e_{n} \in B_{E}$ for $n \in \mathbb{N}$. Then the sequence $\left(w_{n}\right)$ is a linear interpolating sequence for $H_{v_{\alpha}}^{\infty}\left(B_{E}\right)$ and $\mathcal{B}^{\alpha}\left(B_{E}\right)$.

Proof. Let $\left(\alpha_{n}\right)$ be a bounded sequence and define

$$
f(x)=\sum_{n=1}^{\infty} \frac{\alpha_{n}}{z_{n}^{2}} \frac{\left\langle x, e_{n}\right\rangle^{2}}{\left(1-\bar{z}_{n}\left\langle x, e_{n}\right\rangle\right)^{\alpha}} .
$$

The convergence of the series is guaranteed by Bessel's inequality, while the analyticity of $f$ follows from Lemma 1.1. Obviously one has $f\left(w_{m}\right)=\frac{\alpha_{m}}{\left(1-\left|z_{m}\right|^{2}\right)^{\alpha}}$ and also

$$
\left(1-\|x\|^{2}\right)^{\alpha}|f(x)| \leq\left\|\left(\alpha_{n}\right)\right\| \sum_{n=1}^{\infty} \frac{\left|\left\langle x, e_{n}\right\rangle\right|^{2}}{\left|z_{n}\right|^{2}} \frac{\left(1-\|x\|^{2}\right)^{\alpha}}{\left(1-\left|\left\langle x, e_{n}\right\rangle\right|\right)^{\alpha}} \leq(1+\|x\|)^{\alpha} \frac{\left\|\left(\alpha_{n}\right)\right\|}{\inf _{n}\left|z_{n}\right|^{2}}\|x\|^{2} .
$$

This shows that $f \in H_{v_{\alpha}}^{\infty}\left(B_{E}\right)$ and that it interpolates $\left(\alpha_{n}\right)$ at the points $\left(w_{n}\right)$.

Now we turn to the case of $\mathcal{B}^{\alpha}\left(B_{E}\right)$. We define for each bounded sequence $\left(\alpha_{n}\right)$ the functions

$$
g_{\alpha}(x)=\sum_{n=1}^{\infty} \frac{\alpha_{n}\left(1+\left|z_{n}\right|^{2}\right)^{\alpha}}{2(\alpha-1)\left|z_{n}\right|^{4}}\left(\left(1-\bar{z}_{n}^{2}\left\langle x, e_{n}\right\rangle^{2}\right)^{1-\alpha}-1\right), \quad \alpha \neq 1
$$

and

$$
g_{1}(x)=\sum_{n=1}^{\infty} \frac{\alpha_{n}\left(1+\left|z_{n}\right|^{2}\right)}{2\left|z_{n}\right|^{4}} \log \left(\frac{1}{1-\bar{z}_{n}^{2}\left\langle x, e_{n}\right\rangle^{2}}\right) .
$$

To verify that $g_{\alpha}, \alpha \neq 1$, is well defined consider $f_{\alpha}(t)=(1-\lambda t)^{1-\alpha}-1$ for $0 \leq t \leq 1$ and $\lambda \in \mathbb{D}$ and notice that $\left|f_{\alpha}(1)-f_{\alpha}(0)\right| \leq \int_{0}^{1}\left|f_{\alpha}^{\prime}(t)\right| d t \leq \frac{|1-\alpha||\lambda|}{(1-|\lambda|)^{\alpha}}$. An analogous argument works as well for $g_{1}$, by considering $f_{1}(t)=\log (1-\lambda t)$.

Since $\left|\bar{z}_{n}^{2}\left\langle x, e_{n}\right\rangle^{2}\right| \leq\|x\|^{2}<1$, we get that

$$
\left|\left(1-\bar{z}_{n}^{2}\left\langle x, e_{n}\right\rangle^{2}\right)^{1-\alpha}-1\right| \leq \frac{|1-\alpha|\left|\bar{z}_{n}^{2}\left\langle x, e_{n}\right\rangle^{2}\right|}{\left(1-\left|\bar{z}_{n}^{2}\left\langle x, e_{n}\right\rangle^{2}\right|\right)^{\alpha}} \leq \frac{|1-\alpha|\left|\left\langle x, e_{n}\right\rangle\right|^{2}}{\left(1-\left.|| x\right|^{2}\right)^{\alpha}}, \quad \alpha \neq 1
$$

and

$$
\left|\log \left(1-\bar{z}_{n}^{2}\left\langle x, e_{n}\right\rangle^{2}\right)\right| \leq \frac{\left|\bar{z}_{n}^{2}\left\langle x, e_{n}\right\rangle^{2}\right|}{1-\left|\bar{z}_{n}^{2}\left\langle x, e_{n}\right\rangle^{2}\right|} \leq \frac{\left|\left\langle x, e_{n}\right\rangle\right|^{2}}{1-\|x\|^{2}}
$$

Therefore by Bessel's inequality,

$$
\left|g_{\alpha}(x)\right| \leq 2^{\alpha-1}\left\|\left(\alpha_{n}\right)\right\| \sum_{n=1}^{\infty} \frac{\left|\left\langle x, e_{n}\right\rangle\right|^{2}}{\left(1-\|\left. x\right|^{2}\right)^{\alpha}\left|z_{n}\right|^{4}} \leq \frac{2^{\alpha-1}\left\|\left(\alpha_{n}\right)\right\|}{\left(1-\|x\|^{2}\right)^{\alpha} \inf _{n}\left|z_{n}\right|^{4}}\|x\|^{2},
$$

and the analyticity of $g_{\alpha}$ follows again using Lemma 1.1 .

Recall that if $H(x)=h(\langle x, \xi\rangle)$ for a given holomorphic function $h$ in the unit disc then $R H(x)=h^{\prime}(\langle x, \xi\rangle)\langle x, \xi\rangle$. Hence

$$
R g_{\alpha}(x)=\sum_{n=1}^{\infty} \frac{\alpha_{n}}{z_{n}^{2}} \frac{\left(1+\left|z_{n}\right|^{2}\right)^{\alpha}\left\langle x, e_{n}\right\rangle^{2}}{\left(1-\bar{z}_{n}^{2}\left\langle x, e_{n}\right\rangle^{2}\right)^{\alpha}}
$$


Now we obtain

$$
\left(1-\|x\|^{2}\right)^{\alpha}\left|R g_{\alpha}(x)\right| \leq 2^{\alpha}\left\|\left(\alpha_{n}\right)\right\| \sum_{n=1}^{\infty} \frac{\left|\left\langle x, e_{n}\right\rangle\right|^{2}}{\left|z_{n}\right|^{2}} \leq 2^{\alpha} \frac{\left\|\left(\alpha_{n}\right)\right\|}{\inf _{n}\left|z_{n}\right|^{2}}\|x\|^{2} .
$$

Finally since $\left(1-\left\|w_{n}\right\|^{2}\right)^{\alpha} R g_{\alpha}\left(w_{n}\right)=\alpha_{n}$ for all $n \in \mathbb{N}$, the proof is complete.

Also we can rely in the results in one variable to produce examples. Recall that sequences $\left(z_{n}\right) \subset \mathbb{D} \backslash\{0\}$ which are interpolating for any of the spaces we are dealing with in the unit disc satisfy that $\inf _{n}\left|z_{n}\right|>0$. Notice that $\left(z_{n}\right)$ is interpolating for $\mathcal{B}^{\alpha}$ if and only if the mapping $\Upsilon: g \in \mathcal{B}^{\alpha} \rightarrow\left(\left(1-\left|z_{n}\right|^{2}\right)^{\alpha} g^{\prime}\left(z_{n}\right)\right) \in \ell_{\infty}$ is onto.

Proposition 3.4. Let $\alpha>0, \xi \in E$ with $\|\xi\|=1$ and $\left(z_{n}\right) \subset \mathbb{D}$. Denote $w_{n}=z_{n} \xi \in B_{E}$ for $n \in \mathbb{N}$. Then $\left(w_{n}\right)$ is an interpolating sequence for $\mathcal{B}^{\alpha}\left(B_{E}\right)$ if and only if $\left(z_{n}\right)$ is an interpolating sequence for $\mathcal{B}^{\alpha}$.

Proof. Assume that $\left(w_{n}\right)$ is an interpolating sequence for $\mathcal{B}^{\alpha}\left(B_{E}\right)$. Let $\left(\alpha_{n}\right)_{n} \in \ell_{\infty}$ and find $f \in \mathcal{B}^{\alpha}\left(B_{E}\right)$ with $\left(1-\left\|w_{n}\right\|^{2}\right)^{\alpha} R f\left(w_{n}\right)=\alpha_{n}$ for all $n \in \mathbb{N}$. Denote $\varphi(z)=f(z \xi)$. Since $R \varphi(z)=z \varphi^{\prime}(z)=R f(z \xi)$ one has that $\varphi \in \mathcal{B}^{\alpha}$ and we are done since

$$
\left(1-\left|z_{n}\right|^{2}\right)^{\alpha} z_{n} \varphi^{\prime}\left(z_{n}\right)=\left(1-\left\|w_{n}\right\|^{2}\right)^{\alpha} R f\left(w_{n}\right)=\alpha_{n}, \quad n \in \mathbb{N} .
$$

Assume now that $\left(z_{n}\right)$ is an interpolating sequence for $\mathcal{B}^{\alpha}$. Let $\left(\alpha_{n}\right)_{n} \in \ell_{\infty}$ and find $\varphi \in \mathcal{B}^{\alpha}$ with $\left(1-\left|z_{n}\right|^{2}\right)^{\alpha} z_{n} \varphi^{\prime}\left(z_{n}\right)=\alpha_{n}$ for all $n \in \mathbb{N}$. Denote $f(x)=\varphi(\langle x, \xi\rangle)$. Since $R f(x)=$ $\varphi^{\prime}(\langle x, \xi\rangle)\langle x, \xi\rangle$ one has that $f \in \mathcal{B}^{\alpha}\left(B_{E}\right)$ and that it interpolates $\left(\alpha_{n}\right)$ since

$$
\left(1-\left\|w_{n}\right\|^{2}\right)^{\alpha} R f\left(w_{n}\right)=\left(1-\left|z_{n}\right|^{2}\right)^{\alpha} z_{n} \varphi^{\prime}\left(z_{n}\right)=\alpha_{n}, \quad n \in \mathbb{N} .
$$

The analogue to Proposition 3.4 for finite dimensional Hilbert spaces corresponds to the following procedure. Let $L: \mathbb{C}^{n} \rightarrow E$ be an isometric linear embedding and let $P: E \rightarrow \mathbb{C}^{n}$, be the orthogonal projection onto $L\left(\mathbb{C}^{n}\right)$. Then for any $f \in H\left(B_{E}\right)$, and any sequence $\left(z_{m}\right) \subset \mathbb{C}^{n}$, one has

$$
\left(1-\left\|L\left(z_{m}\right)\right\|^{2}\right)^{\alpha} R(f)\left(L\left(z_{m}\right)\right)=\left(1-\left\|z_{m}\right\|^{2}\right)^{\alpha} R(f \circ L)\left(z_{m}\right) .
$$

So we get the following result by also taking into account for $g \in H\left(\mathbb{B}_{n}\right)$ its composition $f=g \circ P \in H\left(B_{E}\right)$.

Remark 3.5. Let $\left(w_{m}\right) \subset B_{E}$ and denote by $E_{0}=\overline{\operatorname{span}\left\{w_{m}: m \in \mathbb{N}\right\}}$. If $\operatorname{dim}\left(E_{0}\right)=n$, consider $\left(z_{m}\right) \subset \mathbb{C}^{n}$ and $L: \mathbb{C}^{n} \rightarrow E_{0}$ the isometry such that $w_{m}=L\left(z_{m}\right)$. Then $\left(z_{m}\right)$ is interpolating for $\mathcal{B}^{\alpha}\left(\mathbb{B}_{n}\right)$ if and only if $\left(w_{n}\right)$ is interpolating for $\mathcal{B}^{\alpha}\left(B_{E}\right)$.

\section{NECESSARY CONDITIONS}

In all the known cases (see $[2,25,19,1])$ a necessary condition for a sequence $\left(z_{n}\right)$ to be interpolating for certain spaces defined in the unit disc $\mathbb{D}$ or the unit ball $\mathbb{B}_{n}$ is to be hyperbolically separated. To extend this result to the case $H_{v}^{\infty}\left(B_{E}\right)$ we shall need a couple of lemmas.

The first one is a Schwarz lemma type consequence of inequality (2.1) in [6] applied to the function $w \in B_{E} \mapsto f(r w)$. 
Lemma 4.1. Let $0<r \leq 1$ and $f: r B_{E} \rightarrow \mathbb{C}$ be a bounded analytic function. Then

$$
|f(x)-f(y)| \leq 2\|f\|_{r B_{E}} \frac{\|x-y\|}{\max \{r-\|x\|, r-\|y\|\}} \text { for all } x, y \in r B_{E},
$$

where $\|f\|_{r B_{E}}=\sup _{\|w\|<r}|f(w)|$.

The second one and its proof are a suitable version of Lemma 14 in [9].

Lemma 4.2. Let $v$ be a weight and $F:[0,1) \rightarrow \mathbb{R}^{+}$be a continuous non decreasing function such that

$$
\frac{v(x)}{v(y)} \leq F\left(\rho_{E}(x, y)\right), \quad x, y \in B_{E}
$$

If $f \in H_{v}^{\infty}\left(B_{E}\right)$ and $0<r<1$ then

$$
|f(x)-f(y)| \leq \frac{4 F(r)}{r v(x)}\|f\|_{v} \rho_{E}(x, y), \quad \text { for } \rho_{E}(x, y) \leq \frac{r}{2} .
$$

The standard weights $v_{\alpha}$, for $\alpha \geq 0$, satisfy the assumption with $F(r)=\left(\frac{4}{1-r^{2}}\right)^{\alpha}$.

Proof. Consider $g=f \circ \varphi_{x}$ and observe that $g$ is an analytic bounded function on $r B_{E}$. Indeed, from (4.1) we have

$$
\left|f\left(\varphi_{x}(z)\right)\right| \leq \frac{\|f\|_{v}}{v\left(\varphi_{x}(z)\right)} \leq \frac{\|f\|_{v}}{v(x)} F\left(\rho_{E}\left(x, \varphi_{x}(z)\right)\right)=\frac{\|f\|_{v}}{v(x)} F(\|z\|) .
$$

Hence $\sup _{\|z\| \leq r}|g(z)| \leq \frac{\|f\|_{v}}{v(x)} F(r)$.

Now applying Lemma 4.1 to $g$, we conclude that for $\left\|\varphi_{x}(y)\right\| \leq r / 2$ we have

$$
\begin{aligned}
|f(x)-f(y)| & =\left|g\left(\varphi_{x}(y)\right)-g\left(\varphi_{x}(x)\right)\right| \\
& \leq 2 \frac{\|f\|_{v}}{v(x)} F(r) \frac{\left\|\varphi_{x}(y)\right\|}{r-\left\|\varphi_{x}(y)\right\|} \\
& \leq \frac{4 F(r)}{r v(x)}\|f\|_{v} \rho_{E}(x, y) .
\end{aligned}
$$

For the final statement, notice that

$$
1-\rho_{E}^{2}(x, y) \leq \frac{\left(1-\|x\|^{2}\right)\left(1-\|y\|^{2}\right)}{(1-\|x\|)^{2}} \leq 2 \frac{1-\|y\|^{2}}{1-\|x\|} \leq 4 \frac{1-\|y\|^{2}}{1-\|x\|^{2}} .
$$

Hence

$$
\frac{v_{\alpha}(x)}{v_{\alpha}(y)} \leq\left(\frac{4}{1-\rho_{E}^{2}(x, y)}\right)^{\alpha}
$$

Theorem 4.3. Let $v$ be a weight satisfying (4.1) for some $F$. Any interpolating sequence $\left(w_{n}\right)$ for $H_{v}^{\infty}\left(B_{E}\right)$ is hyperbolically separated. Further, we have $\inf _{n}\left\{\left\|w_{n}\right\|: w_{n} \neq 0\right\}>0$. 
Proof. Let $M \geq 1$ be an interpolation constant for $\left(w_{n}\right)$. For each $n \in \mathbb{N}$ there exists $f_{n} \in$ $H_{v}^{\infty}\left(B_{E}\right)$ such that $\left\|f_{n}\right\|_{v} \leq M$ and $v\left(w_{m}\right) f_{n}\left(w_{m}\right)=0$ for $m \neq n \in \mathbb{N}$ and $v\left(w_{n}\right) f_{n}\left(w_{n}\right)=1$. Fix $0<r<1$. For $n, m \in \mathbb{N}$ such that $\rho_{E}\left(w_{n}, w_{m}\right) \leq r / 2$, using (4.2) we have

$$
\frac{1}{v\left(w_{n}\right)}=\left|f_{n}\left(w_{n}\right)-f_{n}\left(w_{m}\right)\right| \leq \frac{4 F(r) M}{r v\left(w_{n}\right)} \rho_{E}\left(w_{n}, w_{m}\right) .
$$

In particular if $\rho_{E}\left(w_{n}, w_{m}\right) \leq r / 2$, then $\rho_{E}\left(w_{n}, w_{m}\right) \geq \frac{r}{4 F(r) M}$. Thus we obtain that for any $n, m \in \mathbb{N}$ we have $\rho_{E}\left(w_{n}, w_{m}\right) \geq \min \left\{r / 2, \frac{r}{4 F(r) M}\right\}=\frac{r}{4 F(r) M}$ where the last equality follows since $M F(r) \geq M F(0) \geq M \geq 1$. Hence it is hyperbolically $R$-separated for $R=\sup _{0<r<1} \frac{r}{4 F(r) M}$.

Since for $n \neq m, R \leq \rho_{E}\left(w_{n}, w_{m}\right) \leq \rho_{E}\left(w_{n}, 0\right)+\rho_{E}\left(0, w_{m}\right)=\left\|w_{n}\right\|+\left\|w_{m}\right\|$, no subsequence of $\left(\left\|w_{n}\right\|\right)$ can converge to 0 . Hence $\inf _{n}\left\{\left\|w_{n}\right\|: w_{n} \neq 0\right\}>0$.

Corollary 4.4. Any interpolating sequence for $H_{v_{\alpha}}^{\infty}\left(B_{E}\right), \alpha \geq 0$, is hyperbolically separated.

Example 4.5. Notice that in the one-dimensional case any interpolating sequence $\left(z_{n}\right) \subset \mathbb{D}$ for $\mathcal{B}$ satisfies $\left|z_{n}\right| \rightarrow 1$ when $n \rightarrow \infty$ since it is hyperbolically separated. However, in the infinite dimensional case, there are examples of sequences $\left(w_{n}\right)$ satisfying $\sup _{n \in \mathbb{N}}\left\|w_{n}\right\|<1$ and interpolating for $H_{v}^{\infty}\left(B_{E}\right)$ and $\mathcal{B}^{\alpha}\left(B_{E}\right)$, for instance take $z_{n}=r$ for any fixed $0<r<1$ in Propositions 3.1 and 3.2 .

Now we verify the stability of interpolating sequences under the action of the automorphisms of $B_{E}$.

Lemma 4.6. Let $\varphi: B_{E} \rightarrow B_{E}$ be a holomorphic mapping. Then for $x \in B_{E}$,

$$
\frac{1-\|\varphi(x)\|^{2}}{1-\|x\|^{2}} \geq \frac{1}{2} \cdot \frac{1-\|\varphi(0)\|}{1+\|\varphi(0)\|}
$$

Proof. Recall (see [8, page 48]) that for any analytic self map $\gamma$ of the unit disc, $\frac{1-|\gamma(z)|}{1-|z|} \geq$ $\frac{1-|\gamma(0)|}{1+|\gamma(0)|}$ for all $z \in \mathbb{D}$. Then if $x \neq 0$, we apply this inequality to $\gamma(z)=\left\langle\varphi\left(z \frac{x}{\|x\|}\right), \varphi(x)\right\rangle$ to obtain

$$
\frac{1-\left|\left\langle\varphi\left(z \frac{x}{\|x\|}\right), \varphi(x)\right\rangle\right|}{1-|z|} \geq \frac{1-|\langle\varphi(0), \varphi(x)\rangle|}{1+|\langle\varphi(0), \varphi(x)\rangle|} \geq \frac{1-\|\varphi(0)\|}{1+\|\varphi(0)\|},
$$

so for $z=\|x\|$, we obtain

$$
\frac{1-\|\varphi(x)\|^{2}}{1-\|x\|^{2}} \geq \frac{1-\|\varphi(x)\|^{2}}{2(1-\|x\|)} \geq \frac{1}{2} \cdot \frac{1-\|\varphi(0)\|}{1+\|\varphi(0)\|} .
$$

The estimate is obvious for $x=0$.

Proposition 4.7. Let $v$ be a weight on $B_{E}$ such that for every automorphism $\varphi: B_{E} \rightarrow B_{E}$ there exists $C>0$

$$
C v(\varphi(x)) \geq v(x), \quad x \in B_{E} .
$$

If $\left(w_{n}\right)$ is an interpolating sequence for $H_{v}^{\infty}\left(B_{E}\right)$, then $\left(\varphi\left(w_{n}\right)\right)$ is also an interpolating sequence. The analogous statement holds for $H_{v}^{\infty}\left(B_{E}\right)_{0}$ if $\varphi(0)=0$. The standard weights $v_{\alpha}$ satisfy (4.4). 
Proof. Notice that for $x \in B_{E}$ and $f \in H_{v}^{\infty}\left(B_{E}\right)$ one has $|f(\varphi(x))| \leq \frac{\|f\|_{v}}{v(\varphi(x))} \leq C \frac{\|f\|_{v}}{v(x)}$. This gives $f \circ \varphi \in H_{v}^{\infty}\left(B_{E}\right)$.

Now, given $\left(\alpha_{n}\right) \in \ell_{\infty}$ also the sequence $\left(\frac{v\left(w_{n}\right)}{v\left(\varphi\left(w_{n}\right)\right)} \alpha_{n}\right)$ is bounded. Hence there is $f \in H_{v}^{\infty}\left(B_{E}\right)$ such that $v\left(w_{n}\right) f\left(w_{n}\right)=\frac{v\left(w_{n}\right)}{v\left(\varphi\left(w_{n}\right)\right)} \alpha_{n}$ for all $n \in \mathbb{N}$. Equivalently

$$
v\left(\varphi\left(w_{n}\right)\right) f \circ \varphi^{-1}\left(\varphi\left(w_{n}\right)\right)=\alpha_{n}, \quad n \in \mathbb{N},
$$

that shows that $\left(\varphi\left(w_{n}\right)\right)$ is interpolating for $H_{v}^{\infty}\left(B_{E}\right)$ since $f \circ \varphi^{-1} \in H_{v}^{\infty}\left(B_{E}\right)$.

In order to check that $v_{\alpha}$ satisfies (4.4), just use Lemma 4.6. The remaining statement follows after realizing that now $f \circ \varphi \in H_{v}^{\infty}\left(B_{E}\right)_{0}$ if $f(0)=0$.

Remark 4.8. In the above Proposition 4.7 and the case of $\mathcal{B}\left(B_{E}\right)$, the assumption $\varphi(0)=0$ cannot be avoided. Suppose that the automorphism $\varphi$ transforms interpolating sequences into interpolating sequences. Consider $a=\varphi^{-1}(0)$. If $a \neq 0$, then we may choose in the subspace $\{a\}^{\perp}$ an orthonormal sequence $\left(e_{n}\right)$. Then as in Proposition 3.2, $\left\{a,\|a\| e_{n}: n=1, \ldots\right\}$ is an interpolating sequence for $\mathcal{B}\left(B_{E}\right)$. However, the sequence $\left\{\varphi(a), \varphi\left(\|a\| e_{n}\right): n=1, \ldots\right\}$ is not interpolating since it contains the null vector. Therefore, $0=\varphi(0)$.

\section{SUfFICIENT CONDITIONS}

We begin with a result concerning linear interpolation.

Proposition 5.1. Let $\left(w_{n}\right) \subset B_{E}$ be a linear interpolating sequence for $H_{v_{\alpha}}^{\infty}\left(B_{E}\right), \alpha \geq 0$. Then $\left(w_{n}\right)$ is also linear interpolating for $H_{v_{\beta}}^{\infty}\left(B_{E}\right)$ for any $\beta>\alpha$. If, further, all $w_{n} \neq 0$, then it is linear interpolating for $H_{v_{\beta}}^{\infty}\left(B_{E}\right)_{0}$.

Proof. Since $\left(w_{n}\right)$ is a linear interpolating sequence for $H_{v_{\alpha}}^{\infty}\left(B_{E}\right)$, the corresponding mapping $S$ has a linear right inverse $T$. Put $F_{m}=T\left(e_{m}\right) \in H_{v_{\alpha}}^{\infty}\left(B_{E}\right)$, where $\left(e_{m}\right)_{m}$ is the sequence of canonical unit vectors in $\ell_{\infty}$. Then we have that the sequence $\left(v_{\alpha}\left(w_{n}\right) F_{m}\left(w_{n}\right)\right)_{n}=e_{m}$ and $v_{\alpha}(x) \sum_{m=1}^{\infty}\left|F_{m}(x)\right| \leq\|T\|$ for all $x \in B_{E}$.

Put $\gamma=\beta-\alpha>0$. For each $x \in B_{E}$ we can select $g_{x}(y)=\frac{\left(1-\|x\|^{2}\right)^{\gamma}}{(1-\langle y, x\rangle)^{2 \gamma}}$. It follows that $g_{x} \in H_{v_{\gamma}}^{\infty}\left(B_{E}\right)$ since $v_{\gamma}(y)\left|g_{x}(y)\right| \leq \frac{\left(1-\|y\|^{2}\right)^{\gamma}\left(1-\|x\|^{2}\right)^{\gamma}}{(1-\|x\|)^{\gamma}(1-\|y\|)^{\gamma}} \leq 4^{\gamma}$ and it verifies that $g_{x}(x)=\frac{1}{v_{\gamma}(x)}$.

Define now the operator $\Phi: \ell_{\infty} \rightarrow H_{v_{\beta}}^{\infty}\left(B_{E}\right)$ according to $\Phi\left(\left(\alpha_{n}\right)\right)(x)=\sum_{n=1}^{\infty} \alpha_{n} g_{w_{n}}(x) F_{n}(x)$. It is a well defined and bounded operator since

$$
\sum_{n=1}^{\infty}\left|\alpha_{n} g_{w_{n}}(x) F_{n}(x)\right| v_{\beta}(x) \leq\left\|\left(\alpha_{n}\right)\right\| \sum_{n=1}^{\infty}\left|F_{n}(x)\right| v_{\alpha}(x)\left\|g_{w_{n}}\right\|_{v_{\gamma}} \leq\|T\|\left\|\left(\alpha_{n}\right)\right\| 4^{\gamma} .
$$

Further, $\Phi\left(\left(\alpha_{n}\right)\right)\left(w_{k}\right) v_{\beta}\left(w_{k}\right)=\alpha_{k}$.

To obtain values in $H_{v_{\beta}}^{\infty}\left(B_{E}\right)_{0}$, we can choose the functions $h_{w_{n}}(y)=\frac{\left\langle y, w_{n}\right\rangle}{\left\|w_{n}\right\|^{2}} g_{w_{n}}(y)$ instead of $g_{w_{n}}$, since $\left\|w_{n}\right\|>r$ for some $r>0$ if all $w_{n} \neq 0$.

It is known that if $\left(w_{n}\right) \subset B_{E}$ is interpolating for $H^{\infty}\left(B_{E}\right)$ it is also a linear interpolating sequence (see [21] or [13]). Therefore, we obtain from Proposition 5.1 the following corollary.

Corollary 5.2. If $\left(w_{n}\right) \subset B_{E}$ is an interpolating sequence for $H^{\infty}\left(B_{E}\right)$, then it is linear interpolating for $H_{v_{\alpha}}^{\infty}\left(B_{E}\right)$ for any $\alpha>0$. 
In [11] and [22] it was proved that a sufficient condition for a sequence $\left(w_{n}\right)$ to be interpolating for $H^{\infty}\left(B_{E}\right)$ is to satisfy the Carleson's condition. Hence, we obtain

Corollary 5.3. Let $\left(w_{n}\right) \subset B_{E}$. If there exists $\delta>0$ such that

$$
\prod_{m \neq n} \rho_{E}\left(w_{m}, w_{n}\right) \geq \delta
$$

for all $n \in \mathbb{N}$, then $\left(w_{n}\right)$ is linear interpolating for $H_{v_{\alpha}}^{\infty}\left(B_{E}\right)$.

From Corollary 5.3, we deduce, bearing in mind [12] or $\rho(\|x\|,\|y\|) \leq \rho_{E}(x, y)$, that a sequence $\left(w_{n}\right) \subset B_{E}$ which grows exponentially to the unit sphere, that is, $\frac{1-\left\|w_{n+1}\right\|}{1-\left\|w_{n}\right\|}<c$ for some $c<1$, is linear interpolating for $H_{v_{\alpha}}^{\infty}\left(B_{E}\right)$. In addition, a sequence $\left(w_{n}\right) \subset B_{E}$ such that $\lim _{n \rightarrow \infty}\left\|w_{n}\right\|=1$ has a subsequence which is linear interpolating for $H_{v_{\alpha}}^{\infty}\left(B_{E}\right)$.

In the case $E=\mathbb{C}$ all interpolating sequences in $H_{v_{p}}^{\infty}(\mathbb{D})$ were completely characterized by $\mathrm{K}$. Seip in [25]. Concerning interpolating sequences for $H_{v_{\alpha}}^{\infty}\left(\mathbb{B}_{n}\right)$, let us recall the following results due to X. Massaneda.

Theorem 5.4. ([19, Theorems 1 and 2]). Let $\alpha>0$ and $\left(z_{j}\right) \subset \mathbb{B}_{n}$.

(i) If $\left(z_{j}\right)$ is interpolating for $H_{v_{\alpha}}^{\infty}\left(\mathbb{B}_{n}\right)$, then it satisfies that $K\left(\left\{z_{j}\right\}, \alpha, \beta\right)<\infty$ for any $\beta \geq \alpha$ with $\beta>n$.

(ii) If there exists $\beta \geq \max \{n, \alpha\}$ with $K\left(\left\{z_{j}\right\}, \alpha, \beta\right)<1$, then $\left(z_{j}\right)$ is interpolating for $H_{v_{\alpha}}^{\infty}\left(\mathbb{B}_{n}\right)$.

When replacing $\mathbb{B}_{n}$ by the open unit ball $B_{E}$ of an infinite dimensional Hilbert space $E$, the assumptions in both statements above cannot be anymore fulfilled. Actually Proposition 3.1 shows that interpolating sequences in $H_{v_{\alpha}}^{\infty}\left(B_{E}\right)$ may have $K\left(\left\{w_{j}\right\}, \alpha, \beta\right)=\infty$ for all $\beta>0$.

We now shall see that some of the previously known results can be extended to the infinite dimensional case under the additional assumption of $\eta_{\gamma,\left(w_{n}\right)}$ being a $\gamma$-Carleson measure for some $\gamma$.

Proposition 5.5. Let $\alpha>0$. If $\left(w_{n}\right) \subset B_{E}$ is an interpolating sequence for $H_{v_{\alpha}}^{\infty}\left(B_{E}\right)$, such that $\eta_{\gamma,\left(w_{n}\right)}$ is a $\gamma$-Carleson measure for some $\gamma$, then it is linear interpolating for $H_{v_{\beta}}^{\infty}\left(B_{E}\right)$ for $\beta>\alpha$.

Proof. Let $\left(F_{k}\right) \subset H_{v_{\alpha}}^{\infty}\left(B_{E}\right)$ and $M>0$ such that $F_{k}\left(w_{n}\right)=\frac{\delta_{n}^{k}}{\left(1-\left\|w_{n}\right\|^{2}\right)^{\alpha}}$ and $\left\|F_{k}\right\|_{v_{\alpha}} \leq M$ for all $n, k \in \mathbb{N}$. Put $g_{k}(y)=\frac{\left(1-\left\|w_{k}\right\|^{2}\right)^{\gamma}}{\left(1-\left\langle y, w_{k}\right\rangle\right)^{\beta+\gamma-\alpha}}$. Then $F_{k} \cdot g_{k} \in H_{v_{\beta}}^{\infty}\left(B_{E}\right)$ and the operator $\Phi: \ell_{\infty} \rightarrow H_{v_{\beta}}^{\infty}\left(B_{E}\right)$ defined by $\Phi\left(\left(\alpha_{n}\right)\right)(y)=\sum_{n=1}^{\infty} \alpha_{n} g_{n}(y) F_{n}(y)$ is a well defined and bounded operator since

$$
\begin{aligned}
\sum_{n=1}^{\infty}\left|\alpha_{n} g_{n}(y) F_{n}(y)\right| v_{\beta}(y) & \leq\left\|\left(\alpha_{n}\right)\right\| \sum_{n=1}^{\infty}\left|F_{n}(y)\right|\left(1-\|y\|^{2}\right)^{\alpha} \frac{\left(1-\left\|w_{n}\right\|^{2}\right)^{\gamma}\left(1-\|y\|^{2}\right)^{\beta-\alpha}}{\left|1-\left\langle y, w_{k}\right\rangle\right|^{\beta+\gamma-\alpha}} \\
& \leq M\left\|\left(\alpha_{n}\right)\right\| I_{\eta_{\gamma,\left(w_{n}\right)}}(\beta+\gamma-\alpha, \gamma) .
\end{aligned}
$$

where $I_{\eta_{\gamma,\left(w_{n}\right)}}(\beta+\gamma-\alpha, \gamma)$ is finite because $\eta_{\gamma,\left(w_{n}\right)}$ is a $\gamma$-Carleson measure (Lemma 2.2). To conclude observe that $\Phi\left(\left(\alpha_{n}\right)\right)\left(w_{k}\right)=\frac{\alpha_{k}}{\left(1-\left\|w_{k}\right\|^{2}\right)^{\beta}}$.

Corollary 5.6. ([19, Corollary 1.6]) Let $\alpha>0$. Any interpolating sequence for $H_{v_{\alpha}}^{\infty}\left(\mathbb{B}_{n}\right)$ is linear interpolating for $H_{v_{\beta}}^{\infty}\left(\mathbb{B}_{n}\right)$ for $\beta>\alpha$. 
Proof. It follows by appealing to Theorem 4.3, Proposition 2.7 and choosing $\gamma=n+\alpha$ in Proposition 5.5.

We now give other sufficient conditions for a sequence to be interpolating in this setting that is inspired by the above results and [18, Proposition 1]

Theorem 5.7. Let $\alpha>0$ and $\left(w_{n}\right) \subset B_{E}$ such that $\eta_{\beta,\left(w_{n}\right)}$ is a $\beta$-Carleson measure for some $\beta>0$.

(i) If $\left(w_{n}\right)$ is hyperbolically $R$-separated for some $R>0$ satisfying

$$
\left(1-R^{2}\right)^{\alpha / 2}\left\|\eta_{\beta,\left(w_{j}\right)}\right\|_{\beta}<\frac{2^{\alpha / 2}-1}{2^{\alpha+\beta}}
$$

then it is linear interpolating for $H_{v_{\alpha}}^{\infty}\left(B_{E}\right)$.

(ii) If $K\left(\left\{w_{n}\right\}, \alpha, \beta\right)<1$, then $\left(w_{n}\right)$ is linear interpolating for $H_{v_{\alpha}}^{\infty}\left(B_{E}\right)$.

Proof. For any $\beta+\alpha \geq \epsilon>\beta$ and from Lemma 2.4 we obtain

$$
\sup _{m} \sum_{n=1}^{\infty}\left(1-\rho_{E}\left(w_{m}, w_{n}\right)^{2}\right)^{\epsilon / 2} \frac{\left(1-\left\|w_{n}\right\|^{2}\right)^{\beta-\epsilon / 2}}{\left(1-\left\|w_{m}\right\|^{2}\right)^{\beta-\epsilon / 2}} \leq I_{\eta_{\beta,\left(w_{j}\right)}}(\epsilon, \beta)<+\infty .
$$

Define the operators $S: H_{v_{\alpha}}^{\infty}\left(B_{E}\right) \rightarrow \ell_{\infty}$ given by

$$
S(f)=\left(\left(1-\left\|w_{n}\right\|^{2}\right)^{\alpha} f\left(w_{n}\right)\right)_{n}
$$

and $\Phi: \ell_{\infty} \rightarrow H_{v_{\alpha}}^{\infty}\left(B_{E}\right)$ given by

$$
\Phi\left(\left(\alpha_{n}\right)\right)(x):=\sum_{n=1}^{\infty} \alpha_{n} \frac{\left(1-\left\|w_{n}\right\|^{2}\right)^{2 p-\alpha}}{\left(1-\left\langle x, w_{n}\right\rangle\right)^{2 p}}
$$

for $\left(\alpha_{n}\right) \in \ell_{\infty}$ and $x \in B_{E}$, where $p=\alpha+\beta-\epsilon / 2$. Of course $S$ is a bounded linear operator with $\|S\| \leq 1$.

Since $\beta \leq 2 p-\alpha$ and $\eta_{\beta,\left(w_{n}\right)}$ is a Carleson measure, Lemma 2.4 (iii) for $x=0$, yields that $\sum_{n=1}^{\infty}\left(1-\left\|w_{n}\right\|^{2}\right)^{2 p-\alpha}<\infty$. This guarantees that the series defining $\Phi\left(\left(\alpha_{n}\right)\right)(x)$ is uniformly convergent in $r B_{E}$ for any $0<r<1$ and hence it defines an analytic function on $B_{E}$.

Moreover

$$
\left|\Phi\left(\left(\alpha_{n}\right)\right)(x)\right| \leq\left\|\left(\alpha_{n}\right)\right\| \sum_{n=1}^{\infty} \frac{\left(1-\left\|w_{n}\right\|^{2}\right)^{2 p-\alpha}}{\left|1-\left\langle x, w_{n}\right\rangle\right|^{2 p}} .
$$

or equivalently, since $\epsilon / 2 \leq p$,

$$
\begin{aligned}
\left(1-\|x\|^{2}\right)^{\alpha}\left|\Phi\left(\left(\alpha_{n}\right)\right)(x)\right| & \leq \frac{\left\|\left(\alpha_{n}\right)\right\|}{\left(1-\|x\|^{2}\right)^{p-\alpha}} \sum_{n=1}^{\infty}\left(1-\rho_{E}^{2}\left(x, w_{n}\right)\right)^{p}\left(1-\left\|w_{n}\right\|^{2}\right)^{p-\alpha} \\
& \leq \frac{\left\|\left(\alpha_{n}\right)\right\|}{\left(1-\|x\|^{2}\right)^{\beta-\epsilon / 2}} \sum_{n=1}^{\infty}\left(1-\rho_{E}^{2}\left(x, w_{n}\right)\right)^{\epsilon / 2}\left(1-\left\|w_{n}\right\|^{2}\right)^{\beta-\epsilon / 2} \\
& \leq\left\|\left(\alpha_{n}\right)\right\| I_{\eta_{\beta,\left(w_{j}\right)}}(\epsilon, \beta) .
\end{aligned}
$$

Hence $\Phi$ is well defined and bounded. We aim to prove that $\|I d-S \circ \Phi\|<1$, thus $S \circ \Phi$ will be invertible, hence $S$ has $\Phi \circ(S \circ \Phi)^{-1}$ as right linear inverse. 
INTERPOLATING SEQUENCES FOR WEIGHTED SPACES OF ANALYTIC FUNCTIONS

Consider now the composition operator,

$$
(S \circ \Phi)\left(\left(\alpha_{n}\right)\right)=\left(\left(1-\left\|w_{m}\right\|^{2}\right)^{\alpha} \sum_{n=1}^{\infty} \alpha_{n} \frac{\left(1-\left\|w_{n}\right\|^{2}\right)^{2 p-\alpha}}{\left(1-\left\langle w_{m}, w_{n}\right\rangle\right)^{2 p}}\right)_{m}
$$

and

$$
(I d-S \circ \Phi)\left(\left(\alpha_{n}\right)\right)=-\left(\left(1-\left\|w_{m}\right\|^{2}\right)^{\alpha} \sum_{n=1, n \neq m}^{\infty} \alpha_{n} \frac{\left(1-\left\|w_{n}\right\|^{2}\right)^{2 p-\alpha}}{\left(1-\left\langle w_{m}, w_{n}\right\rangle\right)^{2 p}}\right)_{m} .
$$

We look at the $m^{\text {th }}$ component of $(I d-S \circ \Phi)\left(\left(\alpha_{n}\right)\right)$.

$$
\begin{aligned}
\left.\mid(I d-S \circ \Phi)\left(\left(\alpha_{n}\right)\right)\right)_{m} \mid & \leq\left\|\left(\alpha_{n}\right)\right\|\left(1-\left\|w_{m}\right\|^{2}\right)^{\alpha} \sum_{n=1, n \neq m}^{\infty} \frac{\left(1-\left\|w_{n}\right\|^{2}\right)^{2 p-\alpha}}{\left|1-\left\langle w_{m}, w_{n}\right\rangle\right|^{2 p}} \\
& \leq\left\|\left(\alpha_{n}\right)\right\| K\left(\left\{w_{n}\right\}, \alpha, 2 p-\alpha\right) .
\end{aligned}
$$

Further,

$$
\begin{aligned}
K\left(\left\{w_{n}\right\}, \alpha, 2 p-\alpha\right) & =\sup _{m \in \mathbb{N}} \sum_{n=1, n \neq m}^{\infty}\left(1-\rho_{E}\left(w_{m}, w_{n}\right)^{2}\right)^{p} \frac{\left(1-\left\|w_{n}\right\|^{2}\right)^{p-\alpha}}{\left(1-\left\|w_{m}\right\|^{2}\right)^{p-\alpha}} \\
& \leq \sup _{n, m \in \mathbb{N}}\left(1-\rho_{E}\left(w_{m}, w_{n}\right)^{2}\right)^{\alpha+\beta-\epsilon} K\left(\left\{w_{j}\right\}, \epsilon-\beta, \beta\right) .
\end{aligned}
$$

From this it follows that

$$
\|I d-S \circ \Phi\| \leq I_{\eta_{\beta,\left(w_{j}\right)}}(\epsilon, \beta) \sup _{n, m \in \mathbb{N}}\left(1-\rho_{E}\left(w_{m}, w_{n}\right)^{2}\right)^{\alpha+\beta-\epsilon} \leq I_{\eta_{\beta,\left(w_{j}\right)}}(\epsilon, \beta)\left(1-R^{2}\right)^{\alpha+\beta-\epsilon} .
$$

From (2.2) and picking $\epsilon<\beta+\alpha / 2$ we obtain

$$
I_{\eta_{\beta,\left(w_{j}\right)}}(\epsilon, \beta)\left(1-R^{2}\right)^{\alpha+\beta-\epsilon} \leq\left(1-R^{2}\right)^{\alpha / 2}\left\|\eta_{\beta,\left(w_{j}\right)}\right\|_{\beta} \frac{2^{\frac{\alpha}{2}+\beta}}{1-2^{(\beta-\epsilon)}} .
$$

Since $\inf _{\beta<\epsilon<\beta+\alpha / 2} \frac{1}{1-2^{(\beta-\epsilon)}}=\frac{1}{1-2^{-\alpha / 2}}$ and by choosing $R$ such that

$$
\frac{2^{\frac{\alpha}{2}+\beta}}{1-2^{-\alpha / 2}}\left(1-R^{2}\right)^{\alpha / 2}\left\|\eta_{\beta,\left(w_{j}\right)}\right\|_{\beta}<1
$$

we conclude that $\|I d-S \circ \Phi\|<1$ and therefore $(i)$ is proved.

For $(i i)$, we get from (5.2) that

$$
\|I d-S \circ \Phi\| \leq K\left(\left\{w_{n}\right\}, \alpha, \alpha+2 \beta-\epsilon\right),
$$

for all $\beta<\epsilon \leq \beta+\alpha$. By choosing $\epsilon=\beta+\alpha$, the assumption gives that $\|I d-S \circ \Phi\|<1$ as wanted.

Corollary 5.8. Let $\alpha>0$. If $\left(z_{k}\right) \subset \mathbb{B}_{n}$ is hyperbolically $R$-separated for $R$ close enough to 1 , then it is linear interpolating for $H_{v_{\alpha}}^{\infty}\left(\mathbb{B}_{n}\right)$

Proof. According to Proposition 2.7, $\eta_{\beta,\left(z_{k}\right)}$ is $\beta$-Carleson measure for any $\beta>n$. Then we apply $(i)$ in Theorem 5.7 .

Similarly to the above corollary, we deduce the next result using Corollary 2.9 instead of Proposition 2.7. 
Corollary 5.9. Let $\alpha>0$ and $\left(w_{k}\right) \subset B_{E}$. If the sequence $\left(\left\|w_{k}\right\|\right)$ is hyperbolically $R$-separated for $R$ close enough to 1 , then $\left(w_{k}\right)$ is linear interpolating for $H_{v_{\alpha}}^{\infty}\left(B_{E}\right)$.

\section{Interpolating SEQUENCES FOR RADiAL DERIVATIVES IN Bloch-TyPe SPACES}

Our previous results apply to the Bloch-type spaces by means of the following theorem.

Theorem 6.1. Let $\alpha>0$. The radial derivative mapping

$$
f \in \mathcal{B}^{\alpha}\left(B_{E}\right)_{0} \mapsto R f \in H_{v_{\alpha}}^{\infty}\left(B_{E}\right)_{0}
$$

is an onto isometric isomorphism.

Proof. Notice that for every $m$-homogeneous polynomial $P: E \rightarrow \mathbb{C}$ we have $R P(z)=m P(z)$. Next for any analytic function $f: B_{E} \rightarrow \mathbb{C}$ with Taylor series $f(x)=\sum_{m=0}^{\infty} P_{m}(x)$, its radial derivative $R f(z)=\sum_{m=0}^{\infty} R P_{m}(z)=\sum_{m=1}^{\infty} m P_{m}(z)$, defines an analytic function on $B_{E}$. If moreover, $f \in \mathcal{B}^{\alpha}\left(B_{E}\right)$, then clearly $R f \in H_{v_{\alpha}}^{\infty}\left(B_{E}\right)_{0}$ and $\|f\|_{\mathcal{B}^{\alpha}\left(B_{E}\right)}=\|R f\|_{v_{\alpha}}$, if $f(0)=0$.

To show that the mapping is onto, let $g \in H_{v_{\alpha}}^{\infty}\left(B_{E}\right)_{0}$ with Taylor series $g(x)=\sum_{m=1}^{\infty} Q_{m}(x)$. Define

$$
G(x)=\int_{0}^{1} \frac{g(t x)}{t} d t=\int_{0}^{1} \sum_{m=1}^{\infty} t^{m-1} Q_{m}(x) d t, x \in B_{E} .
$$

The integral exists since the integrand function is a continuous one and $G(0)=0$. Since for every given $x \in B_{E}$ and chosen $1<\lambda$ such that $\|\lambda x\|<1$, the series

$$
\sum_{m=1}^{\infty} \int_{0}^{1} t^{m-1}\left|Q_{m}(x)\right| d t=\sum_{m=1}^{\infty}\left|Q_{m}(\lambda x)\right| \int_{0}^{1} \frac{t^{m-1}}{\lambda^{m}} d t=\sum_{m=1}^{\infty}\left|Q_{m}(\lambda x)\right| \frac{1}{m} \frac{1}{\lambda^{m}},
$$

turns to be convergent, it follows that

$$
G(x)=\sum_{m=1}^{\infty} \int_{0}^{1} t^{m-1} Q_{m}(x) d t=\sum_{m=1}^{\infty} \frac{Q_{m}(x)}{m} .
$$

Hence $G$ is analytic and for its radial derivative $R G(x)=\sum_{m=1}^{\infty} Q_{m}(x)=g(x)$.

Corollary 6.2. Let $\alpha>0$. The sequence $\left(w_{n}\right) \subset B_{E} \backslash\{0\}$ is interpolating for $\mathcal{B}^{\alpha}\left(B_{E}\right)$ if and only if it is interpolating for $H_{v_{\alpha}}^{\infty}\left(B_{E}\right)_{0}$.

Hence the analogous results to Corollary 4.4, Corollary 5.2 and Theorem 5.7 hold for the Bloch-type spaces. Let us state those results in this setting.

Theorem 6.3. Let $\left(w_{n}\right) \subset B_{E} \backslash\{0\}$.

(i) If $\left(w_{n}\right)$ is interpolating for $H^{\infty}\left(B_{E}\right)$, then it is also linear interpolating for $\mathcal{B}^{\alpha}\left(B_{E}\right)$.

(ii) If $\left(w_{n}\right)$ is interpolating for $\mathcal{B}^{\alpha}\left(B_{E}\right)$, then it is hyperbolically $R$-separated for some $R>0$.

(iii) If $\sum_{n=1}^{\infty}\left(1-\left\|w_{n}\right\|^{2}\right)^{2} \delta_{w_{n}}$ is a 2-Carleson measure and $\left(w_{n}\right)$ is hyperbolically $R$-separated for some $R>\sqrt{1-\left(\frac{2^{\alpha / 2}-1}{2^{\alpha+2}\left\|\eta_{2,\left(w_{n}\right)}\right\|_{2}}\right)^{2 / \alpha}}$, then it is linear interpolating for $\mathcal{B}^{\alpha}\left(B_{E}\right)$.

We are interested in producing sequences which are interpolating for $\mathcal{B}$ but not for $H^{\infty}$. Recall that a Blaschke sequence $\left(z_{n}\right) \subset \mathbb{D}$ is a sequence which satisfies $\sum_{k=1}^{\infty}\left(1-\left|z_{k}\right|\right)<\infty$. It is wellknown that if a sequence satisfies the Carleson's condition, then it is a Blaschke sequence. On the other hand, Proposition 2.7 for the case $E=\mathbb{C}$ yields that $\sum_{n=1}^{\infty}\left(1-\left|z_{n}\right|^{2}\right)^{2} \delta_{z_{n}}$ is 2-Carleson 
for any hyperbolically separated sequence $\left(z_{n}\right)$. We will show the existence of a sequence which is hyperbolically $R$-separated for $R$ close enough to 1 which is not a Blaschke sequence. We will adapt the example in [10] to give an example of such a sequence.

Proposition 6.4. Consider $k$ an even number, $k \geq 2$ and circles $C_{n}$ centered at 0 and radius $r_{n}=1-\frac{1}{k^{n}}$ for any $n \geq 1$. In each circle $C_{n}$, we take $z_{n, j}=r_{n} e^{\frac{2 \pi i j}{k^{n-1}}}$ for any $0 \leq j<k^{n-1}$. For any $k \geq 2$, the sequence created in this way is hyperbolically $R_{k}$-separated for some $R_{k}$ so close to 1 as we want. Moreover, the sequence is not a Blaschke sequence.

Proof. Take $z, w$ in the sequence. Then there are two possibilites. If $z$ and $w$ are in different circles $C_{n}$ and $C_{m}, m>n$, then

$$
\rho(z, w) \geq \rho(|z|,|w|)=\frac{r_{m}-r_{n}}{1-r_{m} r_{n}}=\frac{k^{m}-k^{n}}{k^{m}+k^{n}-1} \geq \frac{k^{m-n}-1}{k^{m-n}+1} \geq \frac{k-1}{k+1}
$$

where last inequality is clear since function $\frac{x-1}{x+1}$ is increasing for $x \geq 0$ and $k^{m-n} \geq k$.

Now suppose that $|z|=|w|=r_{n}$. Since $\rho$ is invariant for automorphisms, in particular, for rotations, we consider, without loss of generality, that $z=r_{n}$ and $w=r_{n} e^{\frac{2 \pi i j}{k^{n-1}}}$ for some $1 \leq j \leq \frac{k^{n-1}}{2}$ since we take by symmetry the semicircle $\left\{z \in C_{n}: 0 \leq \arg z \leq \pi\right\}$. Then,

$$
\rho(z, w)=\frac{r_{n}\left|1-e^{\frac{2 \pi i j}{k^{n-1}}}\right|}{\left|1-r_{n}^{2} e^{\frac{2 \pi i j}{k^{n-1}}}\right|}=\frac{2 r_{n} \sin \frac{\pi j}{k^{n-1}}}{\sqrt{\left(1-r_{n}^{2}\right)^{2}+4 r_{n}^{2} \sin ^{2} \frac{\pi j}{k^{n-1}}}}=\frac{1}{\sqrt{1+\left(\frac{1-r_{n}^{2}}{2 r_{n} \sin \frac{\pi j}{k^{n-1}}}\right)^{2}}} .
$$

Bearing in mind that $\sin \alpha \geq \frac{2}{\pi} \alpha$ for any $0 \leq \alpha \leq \frac{\pi}{2}$ and $j \geq 1$, we have that

$$
\rho(z, w) \geq \frac{1}{\sqrt{1+\left(\frac{\left(1-r_{n}^{2}\right) k^{n-1}}{4 r_{n} j}\right)^{2}}} \geq \frac{1}{\sqrt{1+\left(\frac{\left(1-r_{n}^{2}\right) k^{n-1}}{4 r_{n}}\right)^{2}}}=\frac{1}{\sqrt{1+\left(\frac{2 k^{n}-1}{4 k\left(k^{n}-1\right)}\right)^{2}}} .
$$

Hence, for any two terms of the sequence $z, w$ we have that

$$
\rho(z, w) \geq \min \left\{\frac{k-1}{k+1}, \frac{1}{\sqrt{1+\left(\frac{2 k^{n}-1}{4 k\left(k^{n}-1\right)}\right)^{2}}}\right\} .
$$

This expression tends clearly to 1 when $k \rightarrow \infty$ as we wanted. Let $\left(z_{n, j}\right)$ be the sequence that we have constructed. It is clear that it is not a Blaschke sequence since

$$
\sum_{n=1}^{\infty} \sum_{j=0}^{k^{n-1}-1}\left(1-\left|z_{n, j}\right|\right)=\sum_{k=1}^{\infty} k^{n-1}\left(1-r_{n}\right)=\sum_{k=1}^{\infty} k^{n-1} \frac{1}{k^{n}}=\sum_{k=1}^{\infty} \frac{1}{k}=\infty .
$$

The proof is now complete.

Corollary 6.5. There are sequences $\left(z_{k}\right)$ which are interpolating for the Bloch space $\mathcal{B}$ but not interpolating for $H^{\infty}$. This is also valid for the Bloch space $\mathcal{B}\left(B_{E}\right)$ and $H^{\infty}\left(B_{E}\right)$. 
Proof. The first statement is true by Proposition 6.4. The other one follows just by considering the sequence $\left(z_{n} \xi\right)$ where $\left(z_{n}\right)$ is the sequence defined in Proposition 6.4 and $\xi \in E$ such that $\|\xi\|=1$. If $\left(z_{n} \xi\right)$ were interpolating for $H^{\infty}\left(B_{E}\right)$, then $\left(z_{n}\right)$ would be interpolating for $H^{\infty}$ and it is well-known that an interpolating sequence for $H^{\infty}$ satisfies the Carleson's condition and, in particular, it is a Blaschke sequence.

\section{Some Banach Space properties of the Bloch spaCE}

By $\mathcal{B}_{0}\left(B_{E}\right)$ we denote the subspace $\left\{f \in \mathcal{B}\left(B_{E}\right): \lim _{\|x\| \rightarrow 1}\left(1-\|x\|^{2}\right)|R f(x)|=0\right\}$. One can check that if $f \in \mathcal{B}\left(B_{E}\right)$ and $0<r<1$, the function $f_{r}(x):=f(r x)$ defines an element in $\mathcal{B}_{0}\left(B_{E}\right)$.

Lemma 7.1. For every $f \in \mathcal{B}\left(B_{E}\right)$, the net $\left\{f_{r}\right\}_{0<r<1}$ converges to $f$ uniformly on balls of radius less than 1 .

Proof. Firstly we remark that $f$ is bounded on any ball of radius $\sigma<1$, because according to Theorem 3.1 in [4], $|f(x)-f(0)| \leq M \log \frac{1+\|x\|}{1-\|x\|} \leq M \log \frac{1+\sigma}{1-\sigma}$ for some constant $M>0$, independent of $x$.

Fix $0<s<1$ and pick $1>\sigma>s$ and apply Lemma 4.1 to get

$$
\left|f_{r}(x)-f(x)\right|=|f(r x)-f(x)| \leq 2\|f\|_{\sigma B_{E}} \frac{\|r x-x\|}{\sigma-\|x\|} \leq 2\|f\|_{\sigma B_{E}} \frac{1-r}{\sigma-s}
$$

for any $x \in B_{E},\|x\| \leq s$, which shows that $\lim _{r \rightarrow 1} f_{r}(x)=f(x)$ uniformly on $s B_{E}$.

The next result is an extension of [27, Theorem 3.9].

Proposition 7.2. For all $f \in \mathcal{B}\left(B_{E}\right)$, the following estimates hold

$$
\limsup _{\|x\| \rightarrow 1^{-}}\left(1-\|x\|^{2}\right)|R f(x)| \leq d\left(f, \mathcal{B}_{0}\left(B_{E}\right)\right) \leq 2 \limsup _{\|x\| \rightarrow 1^{-}}\left(1-\|x\|^{2}\right)|R f(x)|,
$$

where $d\left(f, \mathcal{B}_{0}\left(B_{E}\right)\right)=\inf \left\{\|f-g\|_{\mathcal{R}\left(B_{E}\right)}: g \in \mathcal{B}_{0}\left(B_{E}\right)\right\}$.

Proof. Since $f_{r} \in \mathcal{B}_{0}\left(B_{E}\right)$, we have $d\left(f, \mathcal{B}_{0}\left(B_{E}\right)\right) \leq\left\|f-f_{r}\right\|_{\mathcal{R}\left(B_{E}\right)}$.

Put $L:=\lim \sup _{\|x\| \rightarrow 1^{-}}\left(1-\|x\|^{2}\right)|R f(x)|$. We seek for an $0<r<1$ such that $\left\|f-f_{r}\right\|_{\mathcal{R}\left(B_{E}\right)}$ is close enough to the upper estimate. Let $\varepsilon>0$, then there is $s<1$ such that $\sup _{\|x\|>s^{\prime}}(1-$ $\left.\|x\|^{2}\right)|R f(x)|<L+\varepsilon$ for all $s^{\prime} \geq s$.

Notice that $R f_{r}(x)=R f(r x)$. Thus for $\|x\|>s$,

$$
\left(1-\|x\|^{2}\right)\left|R f_{r}(x)\right| \leq \frac{1-s^{2}}{1-r^{2} s^{2}}\left(1-\|r x\|^{2}\right)|R f(r x)| .
$$

Since the function $\eta(r)=\frac{1-s^{2}}{1-r^{2} s^{2}}$ has limit 1 when $r \rightarrow 1$, we find $r_{1}<1$ such that for $r \geq r_{1}$, $\frac{1-s^{2}}{1-r^{2} s^{2}} \leq 1+\varepsilon$. And we may choose $r_{1}$ such that also for $\sigma:=\frac{1+s}{2}$ one has $r_{1} \sigma>s$, so that if $\|x\|>\sigma$, then $\|r x\| \geq s$ for all $r \geq r_{1}$. Therefore

$$
\sup _{\|x\| \geq \sigma}\left(1-\|x\|^{2}\right)\left|R f_{r}(x)\right| \leq(1+\varepsilon)(L+\varepsilon) \text { for } r \geq r_{1} .
$$

According to Lemma 4.10 in [4],

$$
\left(1-\|x\|^{2}\right) R f(x)=-\frac{1}{2 \pi i} \int_{|\xi|=1} f\left(\varphi_{x}(\xi x)\right) \frac{d \xi}{\xi^{2}}, x \in B_{E}
$$


which gives the estimate

$$
\left(1-\|x\|^{2}\right)\left|R\left(f-f_{r}\right)(x)\right| \leq \frac{1}{2 \pi} \int_{|\xi|=1}\left|\left(f-f_{r}\right)\left(\varphi_{x}(\xi x)\right)\right| d|\xi| .
$$

It turns out that the set $\left\{\varphi_{x}(\xi x):\|x\|<\sigma,|\xi|=1\right\}$ lies in a ball of radius less than 1 because the $[0,1[$-valued function

$$
\left\|\varphi_{x}(\xi x)\right\|=\left\|\frac{(1-\xi) x}{1-\xi\|x\|^{2}}\right\|=\frac{|1-\xi|\|x\|}{\left|1-\xi\|x\|^{2}\right|}
$$

is a continuous one of the variables $\|x\|$ and $\xi$ on the compact set $[0, \sigma] \times S_{1}$. Now we use Lemma 7.1 to find $r_{0}<1, r_{0}>r_{1}$, such that for $r \geq r_{0}, \sup _{\|x\|<\sigma|\xi|=1}\left|\left(f-f_{r}\right)\left(\varphi_{x}(\xi x)\right)\right| \leq \varepsilon$, and hence, $\sup _{\|x\|<\sigma}\left(1-\|x\|^{2}\right)\left|R\left(f-f_{r}\right)(x)\right| \leq \varepsilon$ for $r \geq r_{0}$. Finally for $r>r_{0}$,

$$
\left\|f-f_{r}\right\|_{\mathcal{R}\left(B_{E}\right)} \leq
$$

$\max \left(\sup _{\|x\|<\sigma,}\left(1-\|x\|^{2}\right)\left|R\left(f-f_{r}\right)(x)\right|, \sup _{\|x\| \geq \sigma}\left(1-\|x\|^{2}\right)|R(f)(x)|+\sup _{\|x\| \geq \sigma}\left(1-\|x\|^{2}\right)\left|R\left(f_{r}\right)(x)\right|\right) \leq$

$$
\max (\varepsilon, L+\varepsilon+(1+\varepsilon)(L+\varepsilon)) .
$$

By letting $\varepsilon \rightarrow 0$, we deduce that $d\left(f, \mathcal{B}_{0}\left(B_{E}\right)\right) \leq 2 \lim \sup _{\|x\| \rightarrow 1^{-}}\left(1-\|x\|^{2}\right)|R f(x)|$.

The estimate $d\left(f, \mathcal{B}_{0}\left(B_{E}\right)\right) \geq \lim \sup _{\|x\| \rightarrow 1^{-}}\left(1-\|x\|^{2}\right)|R f(x)|$ is a routine verification.

Proposition 7.3. The Bloch space $\mathcal{B}\left(B_{E}\right)$ is the dual of

$$
{ }^{*} \mathcal{B}\left(B_{E}\right):=\left\{l \in \mathcal{B}\left(B_{E}\right)^{*}: l \text { is } \tau_{0} \text {-continuous on bounded sets }\right\},
$$

where $\tau_{0}$ denotes the compact-open topology.

Proof. The closed unit ball $\left(U_{\mathcal{B}\left(B_{E}\right)},\|\cdot\|_{\mathcal{B}\left(B_{E}\right)}\right)$ of $\mathcal{B}\left(B_{E}\right)$ is $\tau_{0}$-bounded, since the norm-topology of $\mathcal{B}\left(B_{E}\right)$ is finer than the $\tau_{0}$-topology. Now Montel's theorem ([20, Proposition 9.16]) gives that $U_{\mathcal{B}\left(B_{E}\right)}$ is $\tau_{0}$-relatively compact. If $\left\{f_{\alpha}\right\}$ is a net in $U_{\mathcal{B}\left(B_{E}\right)}$, we conclude that there is a subnet $\left\{f_{\alpha_{j}}\right\}$ such that $f_{\alpha_{j}} \rightarrow f \in H\left(B_{E}\right)$ with respect to the $\tau_{0}$-topology. For $x \in B_{E}$ choose $s>0$ such that $\|x\|+s<1$. Then for any $y \in E$ with $\|y\|=1$, we have by the Cauchy integral formula that

$$
\left\langle y, \overline{\nabla f_{\alpha_{j}}(x)}\right\rangle=\frac{1}{2 \pi i} \int_{|\lambda|=s} \frac{f_{\alpha_{j}}(x+\lambda y)}{\lambda} d \lambda \rightarrow \frac{1}{2 \pi i} \int_{|\lambda|=s} \frac{f(x+\lambda y)}{\lambda} d \lambda=\langle y, \overline{\nabla f(x)}\rangle .
$$

From this we obtain that

$$
|f(0)|+\left(1-\|x\|^{2}\right)|\langle y, \overline{\nabla f(x)}\rangle| \leq \lim _{j}\left\|f_{\alpha_{j}}\right\|_{\mathcal{B}\left(B_{E}\right)} \leq 1
$$

Thus $f \in U_{\mathcal{B}\left(B_{E}\right)}$, i.e. $U_{\mathcal{B}\left(B_{E}\right)}$ is $\tau_{0}$-closed and therefore also $\tau_{0}$-compact. Then ${ }^{*} \mathcal{B}\left(B_{E}\right)$ is a Banach space which is a predual of $\mathcal{B}\left(B_{E}\right)$. In fact, by the Dixmier-Ng theorem [23], $J$ : $\mathcal{B}\left(B_{E}\right) \rightarrow\left({ }^{*} \mathcal{B}\left(B_{E}\right)\right)^{*}, J(f) l:=l(f), f \in \mathcal{B}\left(B_{E}\right), l \in{ }^{*} \mathcal{B}\left(B_{E}\right)$, is an isometric isomorphism.

Proposition 7.4. The space $\mathcal{B}_{0}\left(B_{E}\right)$ is weak* dense in $\mathcal{B}\left(B_{E}\right)$.

Proof. Taking into account that $\left\|f_{r}\right\|_{\mathcal{R}\left(B_{E}\right)} \leq\|f\|_{\mathcal{R}\left(B_{E}\right)}$, the result follows immediately from Lemma 7.1. 
Remark 7.5. The dual space $E^{*}$ is isomorphic to a complemented subspace of $\mathcal{B}\left(B_{E}\right)$. Indeed, the projection mapping $f \in \mathcal{B}\left(B_{E}\right) \stackrel{\pi}{\mapsto} f^{\prime}(0) \in E^{*}$ is continuous since $\left\|f^{\prime}(0)\right\|=\|\nabla f(0)\| \leq$ $\|f\|_{\mathcal{B}\left(B_{E}\right)}$. While on the other hand, the embedding $\mu \in E^{*} \mapsto \mapsto^{\iota} \mu \in \mathcal{B}\left(B_{E}\right)$ is also continuous because for every $x \in B_{E}, \mu^{\prime}(x)=\mu$, so $\sup _{x \in B_{E}}\left(1-\|x\|^{2}\right)\|\nabla \mu(x)\|=\sup _{x \in B_{E}}\left(1-\|x\|^{2}\right)\|\mu\| \leq$ $\|\mu\|$. Finally, $\pi \circ \iota=i d$.

Remark 7.6. Therefore since $E$ is reflexive, neither $\mathcal{B}\left(B_{E}\right)$ nor $\mathcal{B}_{0}\left(B_{E}\right)$ can have the DunfordPettis property. This in sharp contrast to the classical case $(E=\mathbb{C})$ where $\mathcal{B}$ is isomorphic to $\ell_{\infty}$

We close this section with an application of the above results to interpolating sequences.

Proposition 7.7. Let $\left(w_{n}\right) \subset B_{E} \backslash\{0\}$ be a $c_{0}$-linear interpolating sequence for $\mathcal{B}\left(B_{E}\right)$, that $i s$, suppose that there is a linear operator $T: c_{0} \rightarrow \mathcal{B}\left(B_{E}\right)$ such that $S \circ T=i d_{c_{0}}$. Then $\left(w_{n}\right)$ is linear interpolating for $\mathcal{B}\left(B_{E}\right)$.

Proof. Let $\left(e_{m}\right)_{m}$ the sequence of canonical unit vectors in $\ell_{\infty}$. The sequence of functions $T\left(e_{n}\right)=f_{n}$ defines a weakly Cauchy series $\sum_{n} f_{n}$ in $\mathcal{B}\left(B_{E}\right)$ and $\sum_{n}\left|u\left(f_{n}\right)\right| \leq\|T\|\|u\|$ for all $u \in \mathcal{B}\left(B_{E}\right)^{*}$. Hence, for each sequence $\left(\alpha_{n}\right) \in \ell_{\infty}$ the series $\sum_{n} \alpha_{n} f_{n}$ is a weakly Cauchy series in $\mathcal{B}\left(B_{E}\right)$ and $\sum_{n}\left|u\left(\alpha_{n} f_{n}\right)\right| \leq\|T\|\|u\|\left\|\left(\alpha_{n}\right)\right\|$. Thus $\sum_{n} \alpha_{n} f_{n}$ is a $w\left(\mathcal{B}\left(B_{E}\right),{ }^{*} \mathcal{B}\left(B_{E}\right)\right)$ Cauchy series lying in the ball in $\mathcal{B}\left(B_{E}\right)$ of radius $\|T\|$, which is $w\left(\mathcal{B}\left(B_{E}\right),{ }^{*} \mathcal{B}\left(B_{E}\right)\right)$ compact. Hence $\sum_{n} \alpha_{n} f_{n} \in \mathcal{B}\left(B_{E}\right)$ and $\left\|\sum_{n} \alpha_{n} f_{n}\right\|_{\mathcal{R}\left(B_{E}\right)} \leq\|T\|\left\|\left(\alpha_{n}\right)\right\|$.

Define $\tilde{T}: \ell_{\infty} \rightarrow \mathcal{B}\left(B_{E}\right)$ according to $\tilde{T}\left(\left(\alpha_{n}\right)\right)=\sum_{n} \alpha_{n} f_{n}$. Now using (7.1) and recalling that the series also converges for the $\tau_{0}$-topology, we get that

$$
\left(1-\left\|w_{k}\right\|^{2}\right) R\left(\tilde{T}\left(\left(\alpha_{n}\right)\right)\left(w_{k}\right)=\sum_{n} \alpha_{n}\left(1-\left\|w_{k}\right\|^{2}\right) R\left(f_{n}\right)\left(w_{k}\right)=\alpha_{k} .\right.
$$

Thus $(S \circ \tilde{T})=I d_{\ell_{\infty}}$, as wanted.

Acknowledgement. This paper was completed during the 2016 fall semester while Mikael Lindström was visiting Universidad de Valencia whose hospitality is gratefully acknowledged with special thanks to Pablo Galindo. We warmly thank the referees for their very careful reading and the suggestions provided.

\section{REFERENCES}

[1] K. R. M. Attele, Interpolating sequences for the derivatives of the Bloch functions, Glasgow Math. J. 34 (1992), 35-41.

[2] B. Berndtsson, Interpolating sequences for $H^{\infty}$ in the ball, Nederl. Akad. Wetensch. Indag. Math. 47 (1) (1985), 1-10.

[3] O. Blasco, P. Galindo and A. Miralles, Bloch functions on the unit ball of an infinite dimensional Hilbert space, J. Func. Anal. 267 (2014), 1188-1204.

[4] O. Blasco, P. Galindo, M. Lindström and A. Miralles, Composition operators on the Bloch space of the unit ball of a Hilbert space. Banach J. of Math. Anal. 11 (2) (2017), 311-334.

[5] B. Boe and A. Nicolau, Interpolation by functions in the Bloch space. J. Anal. Math. 94 (2004), $171-194$.

[6] T. K. Carne, B. Cole and T. W. Gamelin, A uniform algebra of analytic functions on a Banach space, Trans. Amer. Math. Soc. 314 (2) (1989), 639-659.

[7] B. R. Choe and K. S. Rim, Fractional derivatives of Bloch functions, growth rate and interpolation, Acta Math. Hungar. 72 (1-2) (1996), 67-86. 
[8] C. Cowen and B. MacCluer, Composition operators on spaces of analytic functions, CRC Press, Boca Raton, 1995.

[9] P. Domański and M. Lindström, Sets of interpolation for weighted Banach spaces of holomorphic functions, Annales Polon. Mathematici 79 (3) (2002), 233-264.

[10] P. Duren, A. Schuster and D. Vukotić, On uniformly discrete sequences in the disk, Oper. Theory Adv. Appl. 156 Birkhäuser, Basel, 2005.

[11] P. Galindo, T. W. Gamelin and M. Lindström, Spectra of composition operators on algebras of analytic functions on Banach spaces, Proc. Roy. Soc. Edinb. 139A (2009), 107-121.

[12] P. Galindo and A. Miralles, Interpolating sequences for bounded analytic functions, Proc. Am. Math. Soc. 135 (10) (2007), 3225-3231.

[13] P. Galindo, A. Miralles and M. Lindström, Interpolating sequences on uniform algebras, Topology 48 (2009), 111-118.

[14] J.B. Garnett, Bounded analytic functions. Academic Press, New York, 1981.

[15] D. Girela, J.A. Peláez and D. Vukotić, Uniformly discrete sequences in regions with tangential approach to the unit circle, Complex Var. Elliptic Equ. 52 (2-3) (2007), 161-173.

[16] K. Goebel and S. Reich, Uniform convexity, hyperbolic geometry and nonexpansive mappings, Marcel Dekker, Inc., New York and Basel, 1984.

[17] H. Hamada, Bloch-type spaces and extended Cesàro operators in the unit ball of a complex Banach space, Sci. China Math., to appear.

[18] K. Madigan and A. Matheson, Compact composition operators on the Bloch space, Trans. Amer. Math. Soc. 347 (1995) 2679-2687.

[19] X. Massaneda, $A^{-p}$ interpolation in the unit ball, J. London. Math. Soc. 52 (2) (1995) 391-401.

[20] J. Mujica, Complex analysis in Banach spaces. Dover Books on Mathematics (2010).

[21] J. Mujica, Linearization of holomorphic mappings on infinite-dimensional spaces. X Latin American School of Mathematics (Spanish) (Tanti, 1991). Rev. Un. Mat. Argentina 37 (1-2) (1991), 127-134 (1992).

[22] A. Miralles, Interpolating sequences for $H^{\infty}\left(B_{H}\right)$, Quaest. Math. 39 (6) (2016) 785-795.

[23] K. Ng, On a theorem of Dixmier, Math. Scand. 29 (1971), 279-280.

[24] W. Rudin, Function theory in the unit ball of $\mathbb{C}^{n}$, Springer Verlag, New-York, 1980.

[25] K. Seip, Beurling type density theorems in the unit disk, Invent. Math. 113 (1993), 21-39.

[26] K. Seip, Interpolation and sampling in spaces of analytic functions, University Lecture Series, 33. American Mathematical Society, Providence, RI, 2004.

[27] M. Tjani, Distance of a Bloch function to the little Bloch space, Bull. Aust. Math. Soc. 74 (1), 2006, 101-119.

[28] K. Zhu, Spaces of holomorphic functions in the unit ball, Grad. Texts in Math. 226, Springer Verlag, 2005.

[29] J. Xiao, Carleson measure, atomic decomposition and free interpolation from Bloch space., Ann. Acad. Sci. Fenn. Ser. A I Math. 19 (1) (1994), 35-46.

[30] Z. Xu, Bloch type spaces in the unit ball of a Hilbert space, Preprint

Oscar Blasco. Departamento de Análisis Matemático, Universidad de Valencia, Valencia, SPAIN. e.MAIL: OSCAR.BLASCO@UV.ES

Pablo Galindo. Departamento de Análisis Matemático, Universidad de Valencia, Valencia, SPAIN. e.MAIL: PABLO.GALINDO@UV.ES

Mikael Lindström. Department of Mathematics, Abo Akademi University, Abo, Finland. e.MAIL: MLINDSTR@ABO.FI

Alejandro Miralles. Departament de Matemàtiques and Instituto Universitario de Matemáticas y Aplicaciones de Castellón (IMAC), Universitat Jaume I de Castelló (UJi), Castelló, Spain. e.MAIL: MIRALLEA@UJI.ES 